\title{
LES PAROXYSMES CLIMATO-THERMIQUES EN TUNISIE : APPROCHE MÉTHODOLOGIQUE ET ETUDE DE CAS
}

\author{
Habib BEN BOUBAKER
}

\author{
U.R. Biogéographie, Climatologie Appliquée et Dynamique Erosive \\ Faculté des Lettres, des Arts et des Humanités \\ 2010 Manouba - Tunisie \\ hboubaker@yahoo.fr
}

\begin{abstract}
Résumé
Le climat de la Tunisie, caractérisé par une grande variabilité, est souvent exposé à des extrêmes climatothermiques, parfois aigus, qui méritent le qualificatif de paroxysme. Ces événements extrêmes se manifestent soit par des anomalies thermiques positives très amples, traduisant une chaleur intense, soit par des anomalies négatives, synonymes de paroxysmes de froid. Toutefois, la définition de ces paroxysmes, loin d'être unanime, est objet à controverses. Dans le présent travail, nous proposons une double méthodologie pour déterminer les critères et seuils de ces paroxysmes, adaptée au contexte tunisien. Deux types d'indices de chaleur et de froid sont proposés : relatifs et absolus. Pour valider cette méthodologie, nous avons comme exemple les séries des températures maximales et minimales quotidiennes observées à Tunis-Carthage entre 1950 et 2006.
\end{abstract}

Mots-clés : Paroxysmes thermiques, chaleur, froid, Gumbel, Tunisie.

\begin{abstract}
Climatological-heat paroxysms in Tunisia: methodological approach and case study

The climate of Tunisia, characterized by a great variability, is often exposed to extreme thermal events, which are sometimes acute; therefore, they deserve the qualifier of paroxysm. These extreme events either manifest as positive and very extensive thermal anomalies, marking an intense summer heat, or by important negative anomalies, signalling cold peaks. However, the definitions of these paroxysms, far from being unanimous, are object to controversies. In this work, we propose a methodology to establish the criteria and thresholds of these thermal peaks, adapted to the Tunisian context. Two types of indices of heat and cold are proposed: relative and absolute. To validate this methodology, the series of maximum and minimum daily temperatures observed in the station of Tunis-Carthage during the last half century (1950-2006) can be used as an example.
\end{abstract}

Keywords: Thermal paroxysms, heatwaves, cold waves, Gumbel, Tunisia.

\section{Introduction}

Un paroxysme se définit comme étant «le plus haut degré d'un phénomène » ou une période pendant laquelle un phénomène se manifeste avec le plus d'intensité (Besancenot, 1992 ; dictionnaire Larousse, 1995). S'agissant des paroxysmes climatiques, ils se définissent comme des phénomènes "hors normes ", fortement agressifs à l'encontre de l'organisme humain (Besancenot, 1992, 2001). Les paroxysmes climatiques sont nombreux et variés (inondations, sécheresses, vents violents, canicules, grands froids). Aujourd'hui, on estime qu'ils sont responsables, directement ou indirectement, d'une part importante de décès. Parmi ces paroxysmes, ceux qui sont relatifs à la température attirent l'attention dans le contexte actuel du changement climatique général. L'expression de paroxysmes thermiques est de plus en plus utilisée aujourd'hui par les chercheurs, notamment après les vagues de chaleur mortelles des années 1998, 2003 ou 2006. Ce type de paroxysmes constitue l'un des phénomènes à risque susceptibles de mettre en péril la vie de l'homme et d'avoir des effets néfastes sur la végétation naturelle ou cultivée, la sécurité routière, ... (Ben Boubaker et al., 2004 ; Ben Boubaker, 2006). Ils méritent de ce fait la plus grande attention (Besancenot, 1997).

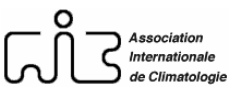


Etudier les paroxysmes thermiques nécessite d'abord de fixer la définition de ce phénomène et surtout, les critères de son identification. Sur ce point, il n'y a toujours pas unanimité entre les chercheurs. Globalement, trois catégories de critères ont été adoptées pour identifier un paroxysme thermique :

i) des seuils de la température, ces seuils variant cependant d'un chercheur à l'autre et d'un type de climat à l'autre ;

ii) les dommages occasionnés par le paroxysme thermique, telle que la surmortalité liée aux vagues de chaleur ;

iii) un double critère associant les deux catégories précédentes. Cette divergence entre les approches se comprend dans la mesure où la température est une notion relative. Une température maximale de $40^{\circ} \mathrm{C}$ peut être considérée normale dans une station saharienne du sud tunisien, mais catastrophique dans un climat tempéré froid. La notion de risque liée aux paroxysmes thermiques est relative aussi. Elle est fonction de plusieurs facteurs socio-économiques, culturels, physiologiques ...

Dans cette étude, nous essayons de donner l'état de la question concernant les critères d'identification des paroxysmes thermiques chauds et froids, en effectuant une analyse critique des seuils thermiques et des indices bioclimatiques qui cherchent à identifier les niveaux de chaleur ou de froid, par référence à des seuils et des critères peu adaptés au contexte tunisien. Nous essayons aussi de proposer une approche méthodologique adaptée au contexte climatique à tendance chaude, en appliquant cette approche à la ville de Tunis.

\section{Les données et les méthodes}

\subsection{Les données}

La température de l'air ambiant est couramment utilisée comme référence pour évoquer soit la chaleur soit le froid. Par définition, cette température est une grandeur physique qui caractérise de façon objective la chaleur ou le froid. Mais leur sensation et leur perception par l'homme restent elles mêmes entachées de subjectivité. De là vient une première difficulté pour identifier les paroxysmes de chaleur et de froid. C'est encore plus compliqué quand il s'agit de définir des chaleurs/froids extrêmes ou paroxysmiques (INVS, 2004). Doit-on se référer à des indicateurs et des seuils statistiques, ou plutôt physiologiques, liés à la "perception humaine» de la chaleur et du froid, elle-même variable d'une ambiance climatique à l'autre, d'un instant à l'autre et d'un individu à l'autre ?

Pour répondre à notre objectif, nous nous focalisons sur les critères thermiques, et nous référons alors aux températures extrêmes maximales diurnes (TX) et minimales nocturnes (TN) observées sur plus d'un demi-siècle à la station de Tunis-Carthage, considérée comme représentative de la ville de Tunis. C'est la seule station qui fournit une série d'observations complète, longue (1950 à 2006), homogène et fiable. Ces données nous ont été gracieusement fournies à partir de la base de données de l'Institut National de la Météorologie. Elles ont été complétées par des observations de cas particuliers disponibles sur le web ${ }^{1}$.

\subsection{Critères d'identification des paroxysmes de froid}

Pour saisir la complexité du problème et orienter notre choix méthodologique, nous avons commencé par effectuer une recherche bibliographique et prospecter l'état de la question sous d'autres climats. Parmi les seuils de froid se référant uniquement à la température, on cite par exemple :

\footnotetext{
${ }^{1}$ www.météo.tn; www.freemeteo.com; http://french.wunderground.com.
} 
- En Belgique, une vague de froid correspond à une période durant laquelle la température minimale journalière reste inférieure à $-2^{\circ} \mathrm{C}$ au moins 7 jours consécutifs et durant laquelle le minimum de cette période descend au moins deux fois sous le seuil des $-7^{\circ} \mathrm{C}$ (Uccle est prise comme station de référence). Cette période peut être accompagnée en tout ou en partie de précipitations hivernales ${ }^{2}$.

- En France, les notions de froid et de grand froid sont couramment utilisées sans qu'il n'existe de définition climatique précise. Les seuils de -5 et $-10^{\circ} \mathrm{C}$ en température minimale sont utilisés comme aide à la décision dans le cadre du plan " urgence hivernale » du Secrétariat à la Lutte contre la Précarité et l'Exclusion, pour mettre en place des mesures d'aide aux personnes sans abri. Les seuils de la vigilance météorologique, plus bas, reposent sur la notion d'indice de refroidissement éolien qui fait intervenir la température et le vent, et sont utilisés pour la population générale.

- En Espagne, l'alerte au froid est donnée dès qu'une descente d'au moins $6^{\circ} \mathrm{C}$ en 24 heures se produit. La température minimale atteinte est ensuite prise en compte et, en fonction de la région considérée, les seuils sont les suivants :

- Température minimale de $0^{\circ} \mathrm{C}$ pour le littoral péninsulaire et celui des îles Baléares ;

- Température minimale entre 0 et $-5^{\circ} \mathrm{C}$ pour des zones situées entre le niveau de la mer et 200 m d'altitude ;

- Température minimale entre -5 et $-10^{\circ} \mathrm{C}$ pour des zones situées entre 200 et $800 \mathrm{~m}$ d'altitude ;

- Température minimale inférieure à $-10^{\circ} \mathrm{C}$ pour des zones situées entre 800 et $1200 \mathrm{~m}$ d'altitude.

Ces critères ne peuvent être appliqués pour la Tunisie et ce, pour plusieurs raisons. D'abord, des températures trop basses, inférieures à $-10^{\circ} \mathrm{C}$ par exemple, n'ont jamais été mesurées en Tunisie. A la station de Thala, la plus élevée et la plus froide en Tunisie, le record absolu des températures minimales nocturnes mesuré (1950-2006) n'est que de $-8,4^{\circ} \mathrm{C}$ (le 9 janvier 1981). Quant aux températures moins basses, de l'ordre de $-5^{\circ} \mathrm{C}$ par exemple, elles sont très rares en Tunisie. Même si elles restent possibles dans les hautes terres intérieures, elles sont impossibles dans les plaines et les régions littorales du pays. Pourtant, dans ces régions, le froid figure parmi les risques redoutés. La population s'y prépare plus ou moins efficacement, proportionnellement aux moyens de chaque foyer (chauffage, couvertures, habillement adéquat, etc.).

Ensuite, pour des questions d'adaptabilité, la sensation de froid est plus précoce à se déclencher chez une population habituée aux hivers doux et aux étés chauds (Eurowinter Group, 1997 ; Keatinge et al., 2000). De ce fait, la population se trouve stressée dès que le mercure s'approche de $0^{\circ} \mathrm{C}$. Même les autorités interviennent pour assister la population en difficulté pour faire face au froid.

D'autres chercheurs, évitant de proposer des seuils thermiques absolus simples de chaleur et de froid, proposent plutôt des indices d'ambiances combinant la température avec un ou plusieurs autres paramètres climatiques. Parmi ces indices, nous citons ceux d'usage fréquent et qui ont été utilisés par des climatologues en Tunisie, malgré certaines critiques (Alouane 2002 ; Henia et Alouane, 2007 et 2009).

- L'indice THI (Temperature Humidity Index) de Thom combine la température et l'humidité de l'air. Il se calcule selon la formule suivante :

\footnotetext{
${ }^{2}$ www.meteobelgique.be
} 


$$
\mathrm{THI}=\mathrm{T}-[(0,55-0,0055 . \mathrm{U}) \cdot(\mathrm{T}-14,5)]
$$

avec $T$ : température de l'air en ${ }^{\circ} \mathrm{C}$, et $U$ : humidité relative en $\%$.

Les ambiances définies par le THI se classent alors selon différents critères (tableau 1).

\begin{tabular}{|c|c|}
\hline THI (Indice de Thom, en ${ }^{\circ} \mathrm{C}$ ) & Ambiance \\
\hline THI $\geq 30$ & Torride \\
\hline 29,9 à 26,5 & Très chaud \\
\hline 26,4 à 20,0 & Chaud \\
\hline 19,9 à 15,0 & Confortable \\
\hline 14,9 à 13 & Frais \\
\hline 12,9 à $-1,7$ & Froid \\
\hline$-1,8$ à $-9,9$ & Très froid \\
\hline THI $\leq-10$ & Extrêmement froid \\
\hline
\end{tabular}

Tableau 1 : Typologie des ambiances biothermiques selon l'indice de Thom (Temperature Humidity Index). Typology of biothermic environments according to the Thom index.

La définition des catégories du froid, telles que proposées par l'indice THI, ne paraissent pas adaptés pour détecter le paroxysme de froid en Tunisie, d'abord parce que le froid est défini dans un éventail très large, avec une marge de $18^{\circ} \mathrm{C}$, allant de -5 à $12,9^{\circ} \mathrm{C}$ (tableau 2 ). Ensuite, entre -1 et $11^{\circ} \mathrm{C}$ (en température absolue), l'indice de Thom présente une variante unique, celle du «froid», quelle que soit l'humidité relative de l'air. On ne passe à l'ambiance supérieure plus rude (très froide) que si la température descend à $-2^{\circ} \mathrm{C}$ et l'humidité relative de l'air (HR) dépasse $90 \%$. Nous jugeons que le seuil de $-2^{\circ} \mathrm{C}$ est trop bas pour convenir au contexte thermique de la Tunisie, particulièrement dans la ville de Tunis, où le record absolu est de $-1,7^{\circ} \mathrm{C}$. Du fait de l'inadaptation de la population locale à des températures très faibles, la sensation du froid, voire du grand froid, est plus rapide à se déclencher, avant même qu'on n'atteigne des températures négatives aussi basses.

Tableau 2 : Valeurs de THI et typologie du froid d'après cet indice de Thom. Values of the THI and typology of cold according the Thom index.

\begin{tabular}{|c|c|c|c|c|c|c|c|c|c|}
\hline Humidité & $-5^{\circ} \mathrm{C}$ & $-4^{\circ} \mathrm{C}$ & $-3^{\circ} \mathrm{C}$ & $-2^{\circ} \mathrm{C}$ & $-1^{\circ} \mathrm{C}$ & $0^{\circ} \mathrm{C}$ & $11^{\circ} \mathrm{C}$ & $12^{\circ} \mathrm{C}$ & $13^{\circ} \mathrm{C}$ \\
\hline $0 \%$ & 5,7 & 6,2 & 6,6 & 7,1 & 7,5 & 8,0 & 12,9 & 13,4 & 13,8 \\
\hline $10 \%$ & 4,7 & 5,2 & 5,7 & 6,2 & 6,7 & 7,2 & 12,7 & 13,2 & 13,7 \\
\hline $20 \%$ & 3,6 & 4,1 & 4,7 & 5,3 & 5,8 & 6,4 & 12,5 & 13,1 & 13,7 \\
\hline $30 \%$ & 2,5 & 3,1 & 3,7 & 4,4 & 5,0 & 5,6 & 12,3 & 13,0 & 13,6 \\
\hline $40 \%$ & 1,4 & 2,1 & 2,8 & 3,4 & 4,1 & 4,8 & 12,2 & 12,8 & 13,5 \\
\hline $50 \%$ & 0,4 & 1,1 & 1,8 & 2,5 & 3,3 & 4,0 & 12,0 & 12,7 & 13,4 \\
\hline $60 \%$ & $-0,7$ & 0,1 & 0,9 & 1,3 & 2,4 & 3,2 & 11,8 & 12,6 & 13,3 \\
\hline $70 \%$ & $-1,8$ & $-0,1$ & $-0,1$ & 0,7 & 1,6 & 2,4 & 11,6 & 12,4 & 13,2 \\
\hline $80 \%$ & $-2,9$ & $-2,0$ & $-1,1$ & $-0,2$ & 0,7 & 1,6 & 11,4 & 12,3 & 13,2 \\
\hline $90 \%$ & $-3,9$ & $-3,0$ & $-2,0$ & $-1,1$ & $-0,1$ & 0,8 & 11,2 & 12,1 & 13,1 \\
\hline $100 \%$ & $-5,0$ & $-4,0$ & $-3,0$ & $-2,0$ & $-1,0$ & 0,0 & 11,0 & 12,0 & 13,0 \\
\hline
\end{tabular}

- L'indice $\mathbf{K}$ de refroidissement éolien (cooling power) est également souvent utilisé en Tunisie (Besancenot, 1986 ; Escourrou, 1994 ; Osczevski, 1995 ; Alouane 2002). Il exprime la quantité de kilocalories (ou watts) perdues par le corps humain par temps froid ou gagnés par temps chaud, par mètre carré de surface corporelle, en une heure. Cet indice $\mathrm{K}$ se calcule comme suit :

$$
\begin{gathered}
\mathrm{K}\left(\mathrm{en} \mathrm{Keal} / \mathrm{m}^{2} / \mathrm{h}\right)=(10.45+10 \sqrt{\mathrm{v-v}})^{*}(33-\mathrm{T}) \\
\mathrm{Ou}: \mathrm{K}\left(\mathrm{en} \mathrm{Watt} / \mathrm{m}^{2}\right)=[1.16 *(10.45+10 \sqrt{\mathrm{v-v}})]^{*}(33-\mathrm{T})
\end{gathered}
$$

avec $\mathbf{v}$ : vitesse du vent en $\mathrm{m} / \mathrm{s}$, et $\mathbf{T}$ : température de l'air en ${ }^{\circ} \mathrm{C}$. 
Cet indice, malgré certains avantages, n'apprécie la température ressentie qu'en rapport avec le vent. Par temps calme, une vitesse du vent nulle neutralise l'équation, d'où la nécessité d'effectuer des corrections ${ }^{3}$. D'autant plus, cet indice est souvent critiqué en raison de la difficulté d'interprétation de ses résultats (Escourrou, 1994; Alouane, 2002). La faiblesse de l'indice $\mathrm{K}$ pourrait s'expliquer également par ses fondements exclusivement physiques (Kunst, 2001 ; Tremblay, 2003).

Pour ces raisons, cet indice est souvent remplacé par un autre assez proche, qui fournit la « température ressentie » dite aussi « windchill factor». Cet indice exprime les seuils de froid par référence à la température ressentie, produit d'une équation combinant la température ambiante et la vitesse du vent. Toutefois, il n'a de sens que si la température est inférieure à $5^{\circ} \mathrm{C}$ et la vitesse du vent supérieure à $5 \mathrm{~km} / \mathrm{h}$. Sinon, Windchill est égal à la température extérieure. Cet indice admet peu de danger (risque faible) pour la santé humaine jusqu'à une température de $-31^{\circ} \mathrm{C}$ avec un vent faible à nul. Le danger (1 minute pour geler la peau) ne commence qu'à partir d'une température de $-32^{\circ} \mathrm{C}$ pour un vent très faible et de $-12^{\circ} \mathrm{C}$ pour un vent soufflant à plus de $32 \mathrm{~km} / \mathrm{h}$ (figure 1). On comprend dès lors qu'un tel indice, même s'il a été amélioré, s'adapte mieux au contexte des climats froids. La nouvelle formule de l'Indice de Refroidissement Eolien (IRE) s'inscrit ainsi (Tremblay, 2003) :

$$
\mathrm{IRE}=13,12+0,6215 . \mathrm{T}-11,37 . \mathrm{V}^{0,16}+0,3965 . \mathrm{T} . \mathrm{V}^{0,16}
$$

avec $T$ la température ambiante en ${ }^{\circ} \mathrm{C}$ et $V$ la vitesse du vent en $\mathrm{km} / \mathrm{h}$.

Figure 1 : Équivalences de température sous le point de congélation. Equivalences of temperature under the freezing point (source: http://www.csao.org/UploadFiles/Manuel/Sante/Chapitre7.pdf).

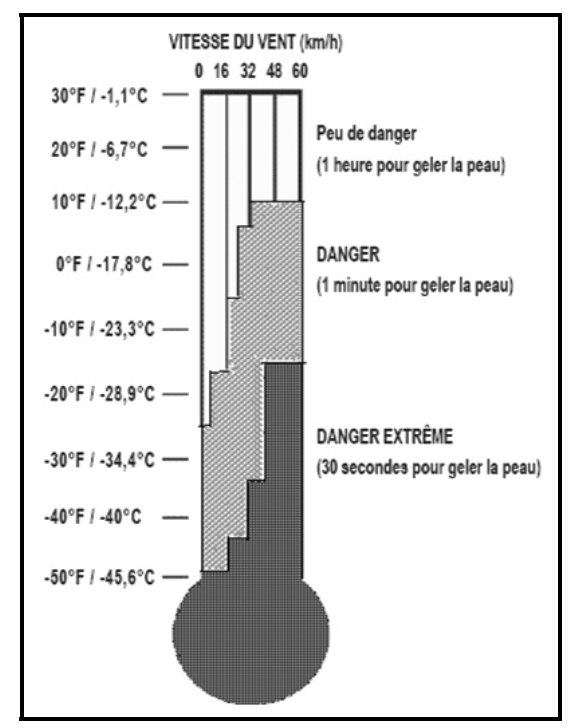

- L'indice Te de Steadman, le plus important développé par cet auteur, est celui de la température apparente, c'est-à-dire la température perçue par un individu (Steadman, 2001). Il se calcule ainsi :

$$
\mathrm{Te}=1,14-1,162 . \mathrm{V}+0,98 . \mathrm{T}+0,0124 \mathrm{~V}^{2}+0,0185 . \mathrm{V} . \mathrm{T}
$$

avec $T$ : température de l'air ambiant en ${ }^{\circ} \mathrm{C}$ et $\mathrm{V}$ : la vitesse du vent en $\mathrm{m} / \mathrm{s}$.

Cet indice est utilisé par le «Meteorological Office » du Royaume-Uni, mais sa méthode est critiquée pour ne pas disposer d'une échelle d'interprétation des valeurs (INVS, 2004).

En somme, nous jugeons qu'aucun des indices bioclimatiques, associant la température à un autre élément du climat, ne peut fournir des seuils objectifs du froid, convenant au contexte climatique de la Tunisie, caractérisée par la douceur relative de son hiver. Ces

\footnotetext{
${ }^{3}$ Généralement, les observations avec une vitesse du vent inférieure à $2 \mathrm{~m} / \mathrm{s}$ sont soit négligées, soit remplacées par la valeur $2 \mathrm{~m} / \mathrm{s}$.
} 
indices se réfèrent à la sensation humaine, elle-même dépendante de facteurs endogènes (âge, genre, acclimatation, etc) et exogènes (saison, latitude, habillement, etc). En outre, le degré de pertinence de ces indices dépend largement du choix des éléments du climat associés à la température (humidité, vent, insolation, etc).

\subsection{Critères d'identification des paroxysmes de chaleur}

La définition des critères des paroxysmes de chaleur n'est pas moins complexe que celle du froid. Une panoplie de définitions et de critères est utilisée pour désigner un épisode de chaleur exceptionnelle, sans qu'il existe une définition consensuelle. Les termes de " canicule », forte/très forte chaleur ou vague de chaleur sont couramment utilisés pour exprimer un épisode de forte chaleur dite exceptionnelle. Nous citons ici quelques exemples de définitions, fondées sur des références purement thermiques de la chaleur :

- L'OMM formule une définition peu explicite de la «vague de chaleur», comme étant "un réchauffement important de l'air, ou une invasion d'air très chaud sur un vaste territoire, généralement de quelques jours à quelques semaines ».

- En France, un jour de chaleur et un autre de forte chaleur correspondent respectivement à une température maximale supérieure ou égale à $25^{\circ} \mathrm{C}$ et $30^{\circ} \mathrm{C}$ (Wahl et al., 2005). Les jours torrides correspondent à des maxima supérieurs à $35^{\circ} \mathrm{C}$. Cette typologie passe sous silence les températures minimales nocturnes. Les paroxysmes de chaleur sont pris en compte par Météo-France et l'INVS pour préparer les bulletins d'alerte. Ces paroxysmes sont définis en rapport avec le risque de surmortalité (INVS, 2003 ; Laaidi et al., 2006). Un indice d'alerte est donc défini pour chaque département, en fonction de l'intensité de la chaleur, mais également du taux de surmortalité pouvant être induit. Certes, ce critère est encore difficile à appliquer en Tunisie, en l'absence d'indicateurs et d'un modèle spécifiant la surmortalité liée au climat.

- En Grande Bretagne, un système de veille sanitaire (heat-health watch) s'installe du $1^{\text {er }}$ juin au 15 septembre. Le seuil de chaleur varie selon les régions, mais il est en moyenne de $30^{\circ} \mathrm{C}$ le jour et de $15^{\circ} \mathrm{C}$ la nuit. Les températures sont jugées «à risque significatif pour la santé » si elles dépassent ces seuils pendant deux jours consécutifs (www.metoffice.gov.uk).

- Aux Etats-Unis, on adopte le seuil de $32,2^{\circ} \mathrm{C}$ comme indicateur de "forte chaleur» (Robinson, 2000 ; Besancenot, 2002 ; INVS, 2003).

En somme, il est délicat de retenir l'un de ces seuils thermiques pour identifier les paroxysmes de chaleur en Tunisie, soit parce que les seuils et les critères manquent de justification, soit parce que les seuils de référence sont très hauts ou très bas, de sorte qu'ils ne correspondent pas au contexte climatique de la Tunisie. Toutefois, certains aspects méthodologiques peuvent être utiles dans une perspective d'élaboration d'un système d'alerte aux excès du climat.

Certains autres chercheurs ont cherché à identifier les catégories de chaleur par référence à des indices plus ou moins complexes, conjuguant la température de l'air à d'autres éléments du climat. En l'occurrence, l'indice THI (Temperature Humidity Index) de Thom permet de calculer la température ressentie par temps chaud, en combinant la température et l'humidité de l'air, exprimée en pourcentages (tableau 3). Cet indice présente également certaines faiblesses à l'égard de la qualification des grandes chaleurs du contexte climato-thermique de la Tunisie. Au-delà d'une température ambiante de $37^{\circ} \mathrm{C}$, chaque degré supplémentaire de chaleur équivaut à un stress additionnel, d'autant plus intense que l'humidité de l'air augmente de quelques points. Or, le THI qualifie l'ambiance de très chaude si : $i$ ) la température de l'air est de $37^{\circ} \mathrm{C}$ et l'humidité relative varie de 15 à $43 \%$; ii) la température 
est de $40^{\circ} \mathrm{C}$ et l'humidité relative de l'air varie de 5 à $30 \%$; iii) la température est de $43^{\circ} \mathrm{C}$ et l'humidité relative de l'air est inférieure à 17\%.

\begin{tabular}{|c|c|c|c|c|c|c|c|c|c|c|}
\hline $\begin{array}{c}\text { Température }\left({ }^{\circ} \mathrm{C}\right) \\
\text { Humidité relative }(\%)\end{array}$ & 27 & 29 & 31 & 34 & 37 & 40 & 43 & 44 & 45 & 47 \\
\hline $\mathbf{0}$ & 20,1 & 21,0 & \begin{tabular}{|l|}
21,9 \\
\end{tabular} & 23,3 & 24,6 & 26,0 & 27,3 & 27,8 & 28,2 & 29,1 \\
\hline 10 & \begin{tabular}{|l|}
20,8 \\
\end{tabular} & 21,8 & \begin{tabular}{|l|}
22,8 \\
\end{tabular} & 24,3 & 25,9 & 27,4 & 28,9 & 29,4 & 29,9 & 30,9 \\
\hline 20 & 21,5 & 22,6 & 23,7 & 25,4 & 27,1 & 28,8 & 30,5 & 31,0 & 31,6 & 32,7 \\
\hline 30 & 22,2 & 23,4 & 24,6 & 26,5 & 28,3 & 30,2 & 32,0 & 32,6 & 33,3 & 34,5 \\
\hline 40 & 22,9 & 24,2 & 25,6 & 27,6 & 29,6 & 31,6 & 33,6 & 34,3 & 34,9 & 36,3 \\
\hline 50 & 23,6 & 25,0 & 26,5 & 28,6 & 30,8 & 33,0 & 35,2 & 35,9 & 36,6 & 38,1 \\
\hline 60 & 24,3 & 25,8 & 27,4 & 29,7 & \begin{tabular}{|l|}
32,1 \\
\end{tabular} & 34,4 & 36,7 & 37,5 & 38,3 & 39,9 \\
\hline 70 & 24,9 & 26,6 & 28,3 & 30,8 & 33,3 & 35,8 & 38,3 & 39,1 & 40,0 & 41,6 \\
\hline 80 & 25,6 & 27,4 & 29,2 & 31,9 & 34,5 & 37,2 & 39,9 & 40,8 & 41,6 & 43,4 \\
\hline 90 & 26,3 & 28,2 & 30,1 & 32,9 & 35,8 & 38,6 & 41,4 & 42,4 & 43,3 & 45,2 \\
\hline 100 & 27,0 & 29,0 & 31,0 & 34,0 & 37,0 & 40,0 & 43,0 & 44,0 & 45,0 & 47,0 \\
\hline 100 & & \multicolumn{2}{|c|}{ Chaud } & & \multicolumn{3}{|c|}{ Très chau } & & \multicolumn{2}{|c|}{ Torride } \\
\hline
\end{tabular}

Tableau 3 : Valeurs de THI et les types d'ambiances chaudes d'après l'indice de Thom. Values of the THI and types of hot environments according to the Thom index.

Le qualificatif « très chaud » reste donc inchangeable dans une marge assez large $\left(\right.$ de $\left.6^{\circ} \mathrm{C}\right)$, variant de $37^{\circ}$ à $43^{\circ} \mathrm{C}$. Dans cette même fourchette ample de $6^{\circ} \mathrm{C}$, le qualificatif de « chaleur torride " reste identique pour une humidité très fluctuante, allant de 44 à 100\%. Par exemple, pour une $\mathrm{HR}$ constante de $50 \%$, l'ambiance produite serait identique (torride), que la température soit de $37^{\circ} \mathrm{C}$, de $43^{\circ} \mathrm{C}$ ou même plus. Autrement dit, dès que la température atteint $30^{\circ} \mathrm{C}$ et l'humidité relative $100 \%$, on entre dans la catégorie torride. Cet intervalle de chaleur mérite donc d'être raffiné. Il ne peut convenir à un pays chaud comme la Tunisie, où des températures supérieures à $40^{\circ} \mathrm{C}$ sont courantes en été. Enfin, les paramètres d'ambiance étant très variables d'un instant à l'autre, le THI ne peut qualifier qu'instantanément une ambiance donnée, en fonction de la température et de l'HR observées simultanément. Cet indicateur se révèle alors insuffisant quand il s'agit de qualifier l'ambiance à l'échelle d'une journée entière (Henia et Alouane, 2007).

L'humidex est un autre indice largement utilisé en saison chaude au Canada. Sans qu'il ne donne de meilleures précisions à l'égard des fortes chaleurs, il permet de distinguer les types d'ambiances associés aux fortes chaleurs. A partir d'une température ressentie de $40^{\circ} \mathrm{C}$, résultant d'une température ambiante de $37^{\circ} \mathrm{C}$ et d'une humidité relative de $25 \%$, il annonce une sensation de malaise assez grande, d'où la recommandation de prudence et de ralentir certaines activités en plein air. Si la température ressentie dépasse $54^{\circ} \mathrm{C}$, le coup de chaleur est jugé imminent. Cette valeur est atteinte à partir d'une température ambiante de $38^{\circ} \mathrm{C}$ associée à une $\mathrm{HR}$ supérieure ou égale à $60 \%$ ou une température de $43^{\circ} \mathrm{C}$ avec $\mathrm{HR} \geq 35 \%$ (www.infoclimat.fr/stations-meteo/expli_humidex.php). Toutefois, malgré certains avantages, cet indice présente au moins deux insuffisances :

- d'abord, il n'a de sens que s'il est calculé pour une température supérieure à $25^{\circ} \mathrm{C}$ et une humidité supérieure à 40\% (sinon, l'Humidex équivaut à la température extérieure) ;

- de cette difficulté découle une seconde, puisque cet indice ne peut être appliqué à tous les mois de l'année. En saison froide, il doit être remplacé par un autre, d'où la perte de précision et la difficulté de comparaison. Le problème se posera également pour les saisons intermédiaires pour lesquelles alternent, en Tunisie, des jours chauds et d'autres froids. Il serait alors question du choix de l'indice le plus pertinent. 
Tout en tenant compte de l'apport de ces différentes tentatives de définition des seuils de chaleur et de froid, ainsi que des différentes critiques formulées, nous proposons d'autres méthodes d'analyse des paroxysmes de chaleur et de froid applicables à la Tunisie.

\section{Analyse des paroxysmes thermiques proposée pour la Tunisie}

\subsection{Approche statistique : des « seuils relatifs » de chaleur et de froid}

\subsubsection{Echelle annuelle}

Face aux difficultés de définition évoquées plus haut et dans le souci de définir des seuils pratiques pour répertorier les paroxysmes de chaleur et de froid, nous avons adopté, en première étape, une démarche statistique. La méthode consiste à analyser les quantiles de la distribution des TX et des TN quotidiennes sur l'ensemble de la série d'observations (19502006) pour calculer le premier quartile (Q1), le premier décile (D1) et le cinquième centile (C5) pour repérer, respectivement, les seuils des jours frais, froids et très froids. Le $3^{\text {ème }}$ quartile (Q3), le $9^{\text {ème }}$ décile (D9) et le $95^{\text {ème }}$ centile (C95) correspondent respectivement aux jours de forte chaleur, de très forte chaleur et aux jours torrides. L'intervalle interquartile représente théoriquement les jours à températures considérées comme ordinaires, proches de la moyenne. Ces derniers représentent les $50 \%$ de la distribution totale ne pouvant être qualifiés comme exceptionnellement chaud ou froid.

Nous avons d'abord considéré l'ensemble de la série des TX et des TN quotidiennes à l'échelle de l'année entière, toutes saisons confondues ; puis ensuite, en séparant les TX et TN quotidiennes, à l'échelle des deux saisons extrêmes, hiver et été. L'analyse des quantiles de la distribution quotidiennes interannuelles (soit 20812 données jours) permet de déduire les seuils de chaleur suivants (tableau 4 et figure 2):

\begin{tabular}{|c|c|c|c|c|c|c|}
\hline & \multicolumn{2}{|c|}{ Année } & \multicolumn{2}{|c|}{ Hiver } & \multicolumn{2}{|c|}{ Eté } \\
\hline & TX & TN & TX & TN & TX & TN \\
\hline $1^{\mathrm{er}}$ quartile & 17,8 & 9 & 14,4 & 5,9 & 29,2 & 18,0 \\
\hline $3^{\text {ème }}$ quartile & 29,5 & 18,5 & 18,1 & 9,6 & 34,1 & 22 \\
\hline $1^{\text {er }}$ décile & 15,1 & 6,4 & 12,6 & 4,3 & 27,2 & 16,2 \\
\hline $9^{\text {ème }}$ décile & 33,2 & 21,5 & 20,1 & 11,3 & 36,7 & 23,6 \\
\hline $5^{\text {ème }}$ centile & 13,7 & 5 & 11,6 & 3,4 & 26 & 15 \\
\hline $95^{\text {ème }}$ centile & 35,2 & 22,8 & 21,5 & 12,2 & 38,4 & 24,5 \\
\hline Maxi absolu & 46,8 & 29,7 & 29,8 & 17,3 & 46,8 & 29,7 \\
\hline Mini absolu & 4,1 & $-1,7$ & 4,1 & $-1,7$ & 16,8 & 9,6 \\
\hline Médiane & 23,2 & 13,3 & 16,2 & 7,7 & 31,7 & 20,0 \\
\hline Moyenne & 23,8 & 13,7 & 16,3 & 7,8 & 31,8 & 20,0 \\
\hline Ecart-type & 7,0 & 3,4 & 3,0 & 2,7 & 3,8 & 2,9 \\
\hline
\end{tabular}

Tableau 4 : Paramètres de sélection $\left(\mathrm{en}^{\circ} \mathrm{C}\right)$ des paroxysmes de chaleur et de froid (TX et TN quotidiennes à Tunis-Carthage, 1950-2006). Parameters of selection $\left({ }^{\circ} \mathrm{C}\right)$ for heat and cold peaks (daily TX and TN in TunisCarthage, 1950-2006).

- les jours de forte chaleur sont compris entre le $3^{\text {ème }}$ quartile $\left(29,5^{\circ} \mathrm{C}\right.$ pour les TX et $18,5^{\circ} \mathrm{C}$ pour les $\left.\mathrm{TN}\right)$ et le $9^{\text {ème }}$ décile $\left(33,2^{\circ} \mathrm{C}\right.$ pour les $\mathrm{TX}$ et $21,5^{\circ} \mathrm{C}$ pour les $\left.\mathrm{TN}\right)$;

- les jours de très forte chaleur correspondent à ceux ayant enregistré des TX entre $33,2^{\circ} \mathrm{C}$ (seuil du $9^{\text {ème }}$ décile) et $35,2^{\circ} \mathrm{C}$ (seuil du $95^{\text {ème }}$ centile) et des TN entre $21,5^{\circ} \mathrm{C}$ et $22,8^{\circ} \mathrm{C}$;

- la chaleur torride correspond aux $5 \%$ des jours ayant enregistré des $\mathrm{TX} \geq 35^{\circ} \mathrm{C}$ et des $\mathrm{TN} \geq 22,8^{\circ} \mathrm{C}$. 
Figure 2 : Echelles de chaleur et de froid correspondantes aux seuils calculés par référence aux TX et $\mathrm{TN}$ quotidiennes, toutes saisons confondues. Scales of heat and cold corresponding to the calculated thresholds by reference to the daily TX and TN, all seasons included.

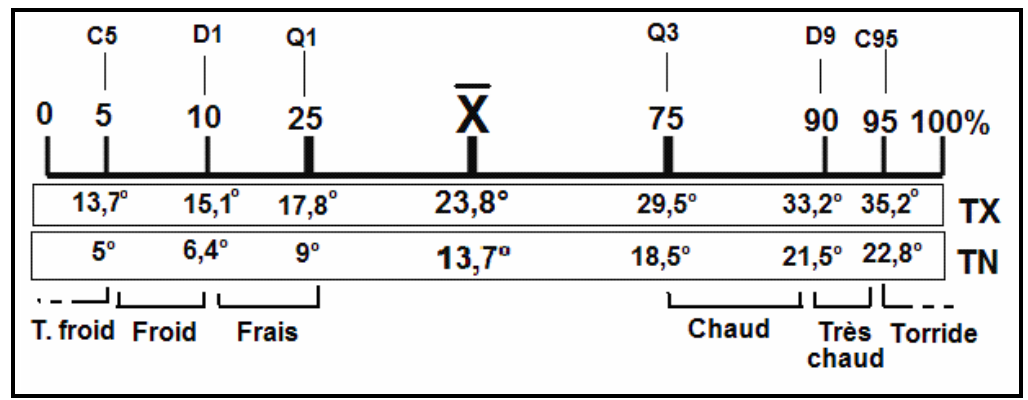

Les gammes de froid déduites de cette première étape d'analyse statistique des quantiles, à l'échelle annuelle permettent de distinguer les trois niveaux suivants :

- les jours frais, avec des TX et TN entre le $1^{\text {er }}$ quartile et le $1^{\mathrm{er}}$ décile, ce qui équivaut à la série entre le $10^{\text {ème }}$ et le $25^{\text {ème }}$ centile de la distribution, soit $15,1<\mathrm{TX} \leq 17,8^{\circ} \mathrm{C}$ et $6,4^{\circ} \mathrm{C}<\mathrm{TN} \leq 9^{\circ} \mathrm{C}$;

- les jours froids, dont les seuils correspondent aux $1^{\text {er }}$ décile $\left(\mathrm{TX}=15,1^{\circ} \mathrm{C}\right.$ et $\left.\mathrm{TN}=6,4^{\circ} \mathrm{C}\right)$ et $5^{\mathrm{eme}}$ centile $\left(\mathrm{TX}=13,7^{\circ} \mathrm{C}\right.$ et $\left.\mathrm{TN}=5^{\circ} \mathrm{C}\right)$;

- les jours très froids, correspondant aux $5 \%$ des températures inférieures au $5^{\text {ème }}$ centile, $c^{\prime}$ est-à-dire avec $\mathrm{TX} \leq 13,7^{\circ} \mathrm{C}$ et $\mathrm{TN} \leq 5^{\circ} \mathrm{C}$.

\subsubsection{Echelle saisonnière}

Les seuils de chaleur sont définis par référence aux TX et TN quotidiennes estivales, soit les mois de juin, juillet et août des 57 années d'observation (1950-2006). L'analyse statistique de cette distribution permet de distinguer trois niveaux de chaleur (figure $3 \mathrm{a}$ ) :

- les jours fortement chauds correspondent aux seuils du Q3 et D9, c'est-à-dire 15\% des jours appartenant à l'intervalle $[34,1 ; 36,7[$ pour les TX $(15 \%$ des jours $)$ et $[22 ; 23,6[$ pour les TN;

- les jours de très forte chaleur correspondent aux jours compris entre le $90^{\text {ème }}$ et le $95^{\text {ème }}$ centile, soit $[36,7 ; 38,4[$ pour les TX et $[23,6 ; 24,5[$ pour les TX ;

- les jours à chaleur torride correspondent aux jours aux températures supérieures au $95^{\text {ème }}$ centile, soit $\mathrm{TX} \geq 38,4^{\circ} \mathrm{C}$ et $\mathrm{TN} \geq 24,5^{\circ} \mathrm{C}$ (rappelons que les limites supérieures des intervalles, correspondant aux records absolus, sont respectivement de $46,8^{\circ} \mathrm{C}$ pour les TX et de $29,7^{\circ} \mathrm{C}$ pour les TN).

Figure 3 : Représentation des seuils relatifs de chaleur correspondant aux $\mathrm{TX}$ et $\mathrm{TN}$ quotidiennes à Tunis : a) pour l'été (juin, juillet et août); b) pour l'hiver (décembre, janvier et février). Diagrammatic representation of the relative thresholds of heat corresponding to the daily $T X$ and $T N$ in Tunis : a) during the summer season (June, July and August) ; b) during the winter season (December, January and February)

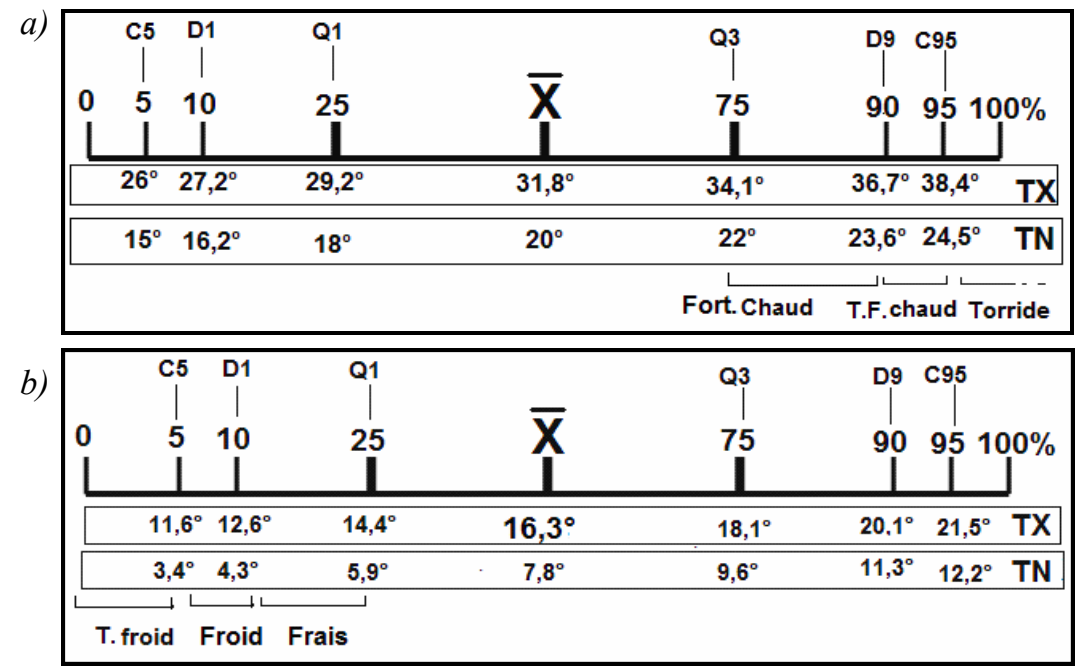


La définition des seuils de froid est élaborée par référence à la série statistique des TX et TN quotidiennes de la saison hivernale (décembre, janvier et février) pour la même période (1950-2006), mais en direction inverse de l'échelle des quantiles $\left(1^{\mathrm{er}}\right.$ quartile, $1^{\mathrm{er}}$ décile, $5^{\text {ème }}$ centile). Les seuils suivants sont identifiés (figure $3 b$ ) :

- les jours frais correspondent à ceux compris entre le $1^{\mathrm{er}}$ quartile et le premier décile, ce qui équivaut aux seuils $12,6^{\circ} \mathrm{C}<\mathrm{TX} \leq 14,4^{\circ} \mathrm{C}$ et $4,3^{\circ} \mathrm{C}<\mathrm{TN} \leq 5,9^{\circ} \mathrm{C}$;

- les jours froids correspondent aux températures comprises entre le $5^{\text {ème }}$ centile (TX $=$ $11,6^{\circ} \mathrm{C}$ et $\left.\mathrm{TN}=3,4^{\circ} \mathrm{C}\right)$ et le $1^{\mathrm{er}}$ décile $\left(\mathrm{TX}=12,6^{\circ} \mathrm{C}\right.$ et $\left.\mathrm{TN}=4,3^{\circ} \mathrm{C}\right)$, soit $5 \%$ des jours inclus dans la distribution statistique hivernale ;

- les jours très froids correspondent aux TX et TN inférieures au $5^{\text {ème }}$ centile, soit TX $\leq$ $11,6^{\circ} \mathrm{C}$ et $\mathrm{TN} \leq 3,4^{\circ} \mathrm{C}$. Les jours très froids ainsi définis à Tunis-Carthage correspondent à l'intervalle $[4,7 ; 11,6]$ pour les TX et $[-1,7 ; 3,4]$ pour les TN.

Toutefois, l'application de ces indices à la sélection des jours de chaleur ou de froid paroxysmique n'est pas aussi simple. Des chevauchements sont toujours possibles entre les différents seuils. En l'occurrence, plusieurs jours peuvent avoir des TN inférieures au 5 ème centile (C5) mais avec des TX supérieures au $10^{\mathrm{eme}}$ centile (D1). Un premier exemple est observé le $12 / 01 / 1985$, avec $\mathrm{TN}<3,4^{\circ} \mathrm{C}$ (très froid) et $11,6 \leq \mathrm{TX}<12,6^{\circ} \mathrm{C}$ (froid). Cette journée a enregistré une température minimale nocturne de $-1,4^{\circ} \mathrm{C}$ et un maximum diurne de $12,2^{\circ} \mathrm{C}$ : faut-il alors privilégier les TN comme critère de froid et faire abstraction des $\mathrm{TX}$ diurnes, ou plutôt trouver le moyen de conjuguer les deux indicateurs ? On citera également l'exemple du 26/01/1992, avec $\mathrm{TN}<3,4^{\circ} \mathrm{C}$ (très froid) et $12,6 \leq \mathrm{TX}<14,4^{\circ} \mathrm{C}$ (frais), et une température minimale nocturne de $0,7^{\circ} \mathrm{C}$ (très froid) et une température maximale diurne de $14^{\circ} \mathrm{C}$ (frais). Pour éviter ce genre de confusions entre les seuils des TX et des TN et rendre les seuils de chaleur et de froid plus pratiques, nous proposons deux grilles de critères (figure 4).

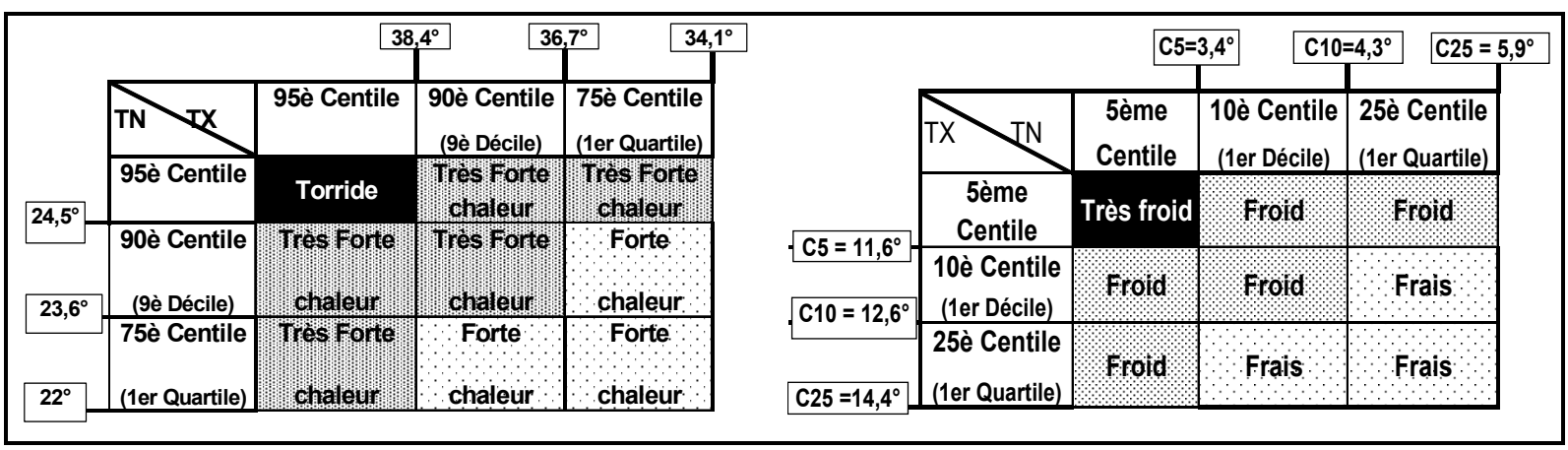

Figure 4 : Grille d'indices de chaleur (à gauche) et de froid (à droite) relatif à Tunis. Indices grid of relative heat (left) and cold (right) in Tunis.

Les critères et seuils relatifs de chaleur et de froid, tels qu'ils sont proposés dans cette démarche saisonnière, représentent des indicateurs plus pertinents et donc, de loin plus pratiques, que ceux intégrant les températures quotidiennes de la totalité de l'année, toutes saisons confondues. Cette approche de définition présente plusieurs points forts. D'abord, elle permet de sélectionner les $25 \%$ des jours les plus chauds et de les partager en trois catégories : chauds, très chauds et torrides. Les $25 \%$ des jours les plus froids sélectionnés s'échelonnent du frais au très froid. Ensuite, cette méthode a l'avantage de prendre en considération le climat thermique saisonnier moyen de la station de Tunis-Carthage comme référence. Cela revient à considérer, implicitement, l'adaptation humaine aux conditions climatiques « ordinaires », puisqu'une température élevée n'est pas ressentie et supportée de la même manière lorsqu'une personne est habituée à vivre dans des conditions de forte chaleur, son organisme ne subissant pas de la même façon le stress thermique. Les modifications du seuil 
de déclenchement de la sudation ainsi que du volume et de la composition de la sueur déterminent le degré d'adaptation d'un individu aux fortes chaleurs. En arrivant dans un environnement chaud, cette personne non habituée à la chaleur éprouve des difficultés à s'adapter et à travailler : sa température corporelle s'élève et une faiblesse physique sévère est possible. Après plusieurs jours, sa tolérance pourrait s'améliorer, avec une augmentation bien moindre de sa température corporelle. Sa sudation débute plus précocement et le volume de sueur qu'elle dégage sera plus important. On considère alors qu'elle est mieux acclimatée à la chaleur (Ganong, 2005). Ces possibilités d'adaptation sont également plus ou moins possibles pour une personne habituée à vivre dans un environnement chaud, et qui se déplace vers un milieu froid.

Enfin, cette approche a l'avantage d'associer simultanément la température de l'air ambiant diurne (TX) et nocturne (TN) comme critères d'identification des paroxysmes thermiques. De cette manière, elle permet de caractériser d'une manière synthétique l'ambiance thermique d'une journée donnée. Pour d'éventuelles applications en matière de gestion des risques liés aux excès de température, prenant en considération l'acclimatation au climat thermique local, cette méthode des seuils saisonniers relatifs trouve une bonne raison d'être. Toutefois, ces seuils « relatifs » de chaleur et de froid extrêmes, tels qu'ils ressortent de l'analyse statistique, si pertinents soient-ils, peuvent poser certains problèmes d'application, en cas de leur extrapolation en dehors de la ville de Tunis. En effet, cette méthode suppose que les seuils calculés pour la station de Tunis-Carthage soient exclusifs. Mais elle ne peut en fait servir à comparer un ensemble de stations appartenant à des topoclimats différents (Ben Boubaker, 2009). Les valeurs des seuils C5 (5 $5^{\text {eme }}$ centile), D1 ( $10^{\text {ème }}$ centile), Q1 $\left(25^{\text {ème }}\right.$ centile), Q3 ( $75^{\text {ème }}$ centile), D9 $\left(90^{\text {ème }}\right.$ centile) et C95 ( $95^{\text {ème }}$ centile) varient d'une station climatique à l'autre, en fonction de la distribution statistique de référence. Il serait délicat de comparer l'intensité de la chaleur ou du froid entre les différents topoclimats et régions climatiques du pays, tant que les valeurs-seuils de la chaleur et du froid ne sont pas identiques. Il faut donc proposer une nouvelle approche, cette fois physiologique et débouchant sur des seuils « absolus », communs pour toutes les stations du pays.

\subsection{Approche physiologique de définition des paroxysmes thermiques : les seuils « absolus » de chaleur et de froid}

\subsubsection{Principes généraux}

Dans cette partie nous cherchons à mettre au point des seuils de chaleur et de froid, reposant sur des critères physiologiques et qui conviennent le mieux au contexte climatothermique de la ville de Tunis. Il est également souhaitable qu'ils soient transposables pour l'ensemble du pays, de manière à permettre la comparaison entre les différentes régions, tout en évitant la ségrégation préjudiciable entre les régions ou les individus. Nous sommes soucieux que ces critères se prêtent à une application aisée, se référant avant tout à la température observée de l'air ambiant, mais sans se contredire avec les indicateurs de la température ressentie, calculée à partir des indices biothermiques qui associent d'autres facteurs d'ambiance, tels que l'humidité de l'air et le vent. Autrement dit, nous distinguons ici deux aspects bien distincts :

- la chaleur elle-même, définie climatiquement par référence à la température de l'air ambiant, ce qui relève de l'objectif de la présente étude ;

- l'impact de la chaleur sur l'organisme humain, pouvant être définie par des indices plus ou moins complexes. L'identification de la chaleur et du froid revient à analyser le bilan radiatif de l'organisme. Cet aspect mérite d'être approfondi séparément, en dissociant les grandes chaleurs des grands froids.

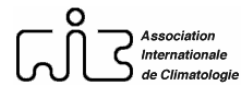


Il est admis que pour un individu ordinaire, il commence à faire chaud dès que l'échange de chaleur entre sa surface corporelle et l'environnement externe aboutit à un gain d'énergie. En revanche, la sensation de froid commence dès qu'il y a une perte. Ces effets de gain et de perte de chaleur dépendent des mécanismes principaux qui assurent l'échange entre le corps et son atmosphère environnante. En l'occurrence, les pertes de chaleur se font pour l'essentiel (à 90\% près) au travers de la peau (Martin et al., 2006) : 3\% par conduction, 15\% par convection, $60 \%$ par radiation et $22 \%$ par évaporation. L'équilibre thermique est obtenu quand la production de chaleur moins les pertes par rayonnement, conduction, convection et évaporation (sudation, respiration) est égale à zéro. Lors d'un paroxysme de chaleur, le corps va tenter d'équilibrer son bilan thermique grâce aux réponses comportementales de l'hypothalamus ${ }^{4}$ et ce, par plusieurs mécanismes :

- en augmentant les échanges, essentiellement par radiation et convection, par le biais d'une vasodilatation cutanée, entraînant une élévation de la température au niveau de la peau. Cette redistribution du débit sanguin peut être possible par une augmentation du débit cardiaque. Toutefois, lorsque la température extérieure dépasse $35^{\circ} \mathrm{C}$, ce mécanisme n'est plus efficace ${ }^{5}$;

- en augmentant l'évaporation de la sueur, à condition qu'elle ne soit pas entravée par des vêtements ou le ruissellement sur la peau par exemple. Mais ce phénomène peut être limité par une perte sudorale maximale.

Toutefois, si l'équilibre thermique entre la quantité de chaleur produite et celle perdue est rompu (bilan excédentaire), les systèmes thermorégulateurs de l'organisme sont menacés de défaillance, avec un risque d'hyperthermie. Elle pourrait provoquer des dysfonctions neurologiques et une dénaturation des protéines, d'où une mortalité instantanée ou retardée. La médecine admet que la température critique chez l'homme est proche de $43^{\circ} \mathrm{C}$. Vers 49 à $50^{\circ} \mathrm{C}$, toutes les structures cellulaires sont détruites et la mort cellulaire survient en moins de 5 minutes (Martin et al., 2006). En revanche, dans le cas de paroxysmes de froid, les sujets, surtout les plus vulnérables (enfants et vieillards), sont menacés d'hypothermie. Dans la littérature médicale, trois phases de gravité croissante sont retenues, en rapport avec la température corporelle : hypothermie mineure $\left(32\right.$ à $\left.35^{\circ} \mathrm{C}\right)$, modérée $\left(28\right.$ à $\left.32^{\circ} \mathrm{C}\right)$ et sévère $\left(<28^{\circ} \mathrm{C}\right)$. Dans le cas d'hypothermie, le corps réagit par plusieurs mécanismes dont l'augmentation du métabolisme basal, une vasoconstriction cutanée, des frissons, ...

Bien qu'il s'agisse de la température corporelle, ces seuils de chaleur et de froid évoqués plus haut sont à prendre en considération dans l'établissement des seuils des paroxysmes thermiques, par référence à la température de l'air ambiant. La zone thermiquement neutre de l'air ambiant admise en médecine, pour un sujet dénudé, s'étend entre 25 et $30^{\circ} \mathrm{C}$ (Widmaier et al., 2007). A des températures ambiantes plus basses, même une vasoconstriction maximale ne peut empêcher une perte de chaleur excessive par rapport à sa production. Donc, il doit exister une augmentation de la production de chaleur pour maintenir la température. A des températures ambiantes supérieures à la zone thermiquement neutre, même une vasodilatation maximale ne peut assurer une perte de chaleur équivalente à sa production. Un autre mécanisme d'élimination de la chaleur (la sudation) entre alors en fonctionnement. A des températures ambiantes dépassant celles du corps, il y a addition de chaleur de l'organisme par radiation et conduction. L'évaporation est alors le seul mécanisme d'évacuation

\footnotetext{
${ }^{4}$ Le centre de thermorégulation est situé au niveau de la région préoptique de l'hypothalamus antérieur. Les informations y sont intégrées sous forme d'une température corporelle moyenne qui est comparée à une zone de températures de référence (zone de neutralité thermique) au-dessus ou en dessous de laquelle les réponses thermorégulatrices sont déclenchées (Martin et al., 2006).

${ }^{5}$ http://infodoc.inserm.fr/doc/ThemCaniculePubli.nsf/ (consulté le 7-3-2009).
} 
thermique. La capacité d'une personne à tolérer de fortes chaleurs dépend donc de l'humidité de l'air et des possibilités de sudation maximale. On comprend donc que les caractéristiques biothermiques de l'air ambiant sont déterminantes pour le confort ou le stress de l'organisme, constamment soumis à cet air, de jour comme de nuit. Même l'ambiance qui règne à l'intérieur des bâtiments, si artificialisée soit-elle, n'échappe pas à ces effets, quelle que soit l'heure de la journée.

Mais, comment procéder pour établir des seuils physiologiques conjuguant les critères de la température corporelle et celle de l'air ambiant ? Le premier principe à considérer, pour définir les paroxysmes de chaleur ou de froid, consiste à valoriser conjointement les TX diurnes et les TN nocturnes. Un paroxysme de froid, défini uniquement par référence à des TN négatives, peut être faussé par des températures diurnes suffisamment élevées pour permettre à l'organisme de récupérer de la chaleur pendant le jour (par exemple, le $04 / 02 / 1989$ à Tunis, avec $\mathrm{TN}=-0,3^{\circ} \mathrm{C}$ et $\mathrm{TX}=16,7^{\circ} \mathrm{C}$ ). En revanche, des $\mathrm{TN}$ nocturnes positives, légèrement supérieures à $0^{\circ} \mathrm{C}$, relayées par des TX diurnes assez faibles (par exemple, le $31 / 01 / 1999$ à Tunis, avec $\mathrm{TN}=1^{\circ} \mathrm{C}$ et $\mathrm{TX}=5,6^{\circ} \mathrm{C}$ ), peuvent soumettre l'organisme à un stress beaucoup plus intense et plus persistant.

Le second principe consiste à assurer le meilleur compromis entre la température ressentie et celle de l'air ambiant, comme critères de définition des paroxysmes thermiques. Pour ce faire, nous avons également testé plusieurs indices bioclimatiques d'usage courant (cf. §1.2 et 1.3). Après plusieurs tâtonnements, il s'avère que ces indices ne se prêtent pas à l'analyse simultanée de la chaleur et du froid, avec la même performance et à l'échelle des différentes saisons thermiques de l'année. En l'occurrence, Météo-Canada se réfère à l'indice de refroidissement éolien (Windchill) pour estimer le froid en hiver pour une température inférieure à $5^{\circ} \mathrm{C}$ et une vitesse du vent supérieure à $5 \mathrm{~km} / \mathrm{h}$ et à l'indice Humidex (Heat index) pour estimer la chaleur en été pour une température de l'air supérieure à $27^{\circ} \mathrm{C}$ et une humidité supérieure à $40 \%{ }^{6}$. Ensuite, les paramètres d'ambiance associés aux grandes chaleurs ou aux grands froids sont très variables d'un moment à l'autre et leur mesure est instantanée. Par jour de très forte chaleur par exemple, le stress peut s'accentuer l'après-midi, avec l'advection d'un air marin humide ou, au contraire, s'apaiser si un vent modéré souffle quelques instants. Les ambiances vécues quelques moments ne peuvent être extrapolées à l'ensemble de la journée. Enfin, en l'absence d'un système d'alerte aux canicules et aux grands froids en Tunisie, l'usage des indices bioclimatiques comme outil pour définir les chaleurs ou les froids paroxysmiques n'est pas à la portée de l'utilisateur. D'autant plus, pour pouvoir juger si une journée donnée est trop chaude ou trop froide, l'usager est appelé à collecter plusieurs indicateurs et à les conjuguer via des indices, souvent sujets à critiques et sans cesse améliorés. Cette tâche n'est évidemment pas à la portée d'un non-spécialiste.

Compte tenu de ces difficultés et conformément à notre objectif initial, nous nous contentons de définir ci-après, des seuils climato-thermiques plus simples de chaleur et de froid, basés uniquement sur les températures maximales diurnes et minimales nocturnes de l'air. Ils se réfèrent aux normes physiologiques courantes et non à la température ressentie, calculée par les indices.

\subsubsection{Approche physiologique de la définition des paroxysmes de chaleur}

\subsubsection{Les seuils " absolus " de chaleur}

D'après les principes physico-thermiques évoqués plus haut, plusieurs seuils physiologiques corporels peuvent être déduits. Le seuil de $33^{\circ} \mathrm{C}$ représente la température

\footnotetext{
${ }^{6}$ http://www.meteo-courgeon.com/humidex-windchill.html
} 
ordinaire de la peau (Vigneau, 2000). Si la température de l'air ambiant est plus élevée, le corps commence à éprouver une sensation de chaleur, abstraction faite des autres facteurs d'ambiance. Le stress physiologique augmente proportionnellement au dépassement de ce seuil, en particulier si la température ambiante franchit $37^{\circ} \mathrm{C}$, température vitale pour l'organisme humain, au-dessus de laquelle une hyperthermie peut devenir critique pour la santé. Le seuil de $40^{\circ} \mathrm{C}$ de température centrale du corps définit le risque de «coup de chaleur » au sens médical du terme (Martin et al., 2006). Chez certains sujets, des convulsions apparaissent dès le franchissement de ce seuil. Le risque devient encore plus grave quand la température corporelle devient supérieure ou égale à $43^{\circ} \mathrm{C}$. C'est le seuil critique chez l'homme et la limite absolue de sa survie (Widmaier et al., 2007). Ce seuil dépassé, les cellules vitales risquent la mort. Il est donc certain que l'exposition (par la peau et/ou la respiration) à des températures de l'air ambiant supérieures à $40^{\circ} \mathrm{C}$ représente un risque grave pour la santé. Il est enfin nécessaire de rappeler qu'une température minimale nocturne de l'air ambiant de $20^{\circ} \mathrm{C}$ est couramment jugée comme valeur seuil au-dessus de laquelle une nuit est jugée à chaleur « tropicale ».

Compte tenu de ces seuils, nous proposons une grille des niveaux absolus de chaleur (figure 5). Les seuils sont définis en additionnant les TX diurnes et les TN nocturnes. Ils s'échelonnent par des pas de $6^{\circ} \mathrm{C}$, partant de $33^{\circ} \mathrm{C}$ pour les premières et de $20^{\circ} \mathrm{C}$ pour les secondes. Trois principales catégories de fortes chaleurs sont alors distinguées :

- les jours de forte chaleur correspondent à une somme $\mathrm{TX}+\mathrm{TN}>53^{\circ} \mathrm{C}$, avec $\mathrm{TX}>$ $33^{\circ} \mathrm{C}$ et $\mathrm{TN} \geq 20^{\circ} \mathrm{C}$;

- les jours de très forte chaleur correspondent à une somme TX+TN comprise entre 60 et $66^{\circ} \mathrm{C}$;

- les jours torrides correspondent à une somme des $\mathrm{TX}+\mathrm{TN}$ supérieure ou égale à $67^{\circ} \mathrm{C}$.

\begin{tabular}{|c|c|c|c|c|c|c|c|c|c|c|c|c|c|c|c|c|}
\hline TIN & $33^{\circ} \mathrm{C}$ & $34^{\circ} \mathrm{C}$ & $35^{\circ} \mathrm{C}$ & $36^{\circ} \mathrm{C}$ & $37^{\circ} \mathrm{C}$ & $38^{\circ} \mathrm{C}$ & $39^{\circ} \mathrm{C}$ & $40^{\circ} \mathrm{C}$ & $41^{\circ} \mathrm{C}$ & $42^{\circ} \mathrm{C}$ & $43^{\circ} \mathrm{C}$ & $44^{\circ} \mathrm{C}$ & $45^{\circ} \mathrm{C}$ & $>=46^{\circ} \mathrm{C}$ & & \\
\hline $20^{\circ} \mathrm{C}$ & 53 & 54 & 55 & 561 & 57 & 58 & 59 & 60 & 61 & 62 & 63 & 64 & 65 & 66 & & \\
\hline $21^{\circ} \mathrm{C}$ & 54 & 55 & 56 & ji & 58 & 59 & 60 & 61 & 8 & 13 & 64 & 65 & 66 & 67 & & \\
\hline $22^{\circ} \mathrm{C}$ & 55 & 56 & ais & 58 & 59 & 60 & 61 & 8 & & 64 & 65 & 66 & 67 & 68 & & \\
\hline $23^{\circ} \mathrm{C}$ & 56 & Sis & 58 & 59 & 60 & 61 & 67 & 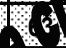 & 4 & 65 & 66 & 57 & ( & 69 & & \\
\hline $24^{\circ} \mathrm{C}$ & 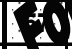 & 5 & 59 & 60 & 61 & 62 & 3 & 64 & 65 & 66 & 67 & & & 70 & & \\
\hline $25^{\circ} \mathrm{C}$ & 58 & 59 & 60 & 61 & 62 & 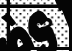 & 84 & 65 & 66 & 67 & & & 70 & 71 & Légende & \\
\hline $26^{\circ} \mathrm{C}$ & 59 & 60 & 61 & 62 & 61 & 8 & 65 & 66 & 67 & 69 & 10 & 70 & 71 & 72 & Somme $T X$ et $T N$ & Journée \\
\hline $27^{\circ} \mathrm{C}$ & 60 & 61 & 62 & 63 & 64 & 65 & 66 & 67 & & a) & 70 & 71 & 72 & 73 & $>=67^{\circ} \mathrm{C}$ & Torride \\
\hline $28^{\circ} \mathrm{C}$ & 61 & 62 & 63 & 64 & 65 & 66 & 67 & 68 & & 70 & 71 & 72 & 73 & 74 & $60 \% 66 \%$ & Très forte chaleur \\
\hline$>=29^{\circ} \mathrm{C}$ & 62 & 63 & 64 & 65 & 66 & 67 & 68 & 69 & 10 & 71 & 72 & 73 & 74 & 75 & $53^{\circ}$ à $59^{\circ} \mathrm{C}$ & Forte chaleur \\
\hline
\end{tabular}

Figure 5 : Seuils absolus de chaleur et typologie des jours fortement chauds, très fortement chauds et torrides à Tunis. Thresholds and types of absolute heat in highly-hot, extremely-hot and scorching-hot days in Tunis.

Nous pouvons déduire que la typologie des fortes chaleurs, telle que proposée ci-dessus, contribue à affiner et à simplifier celle élaborée à partir des indices THI et Humidex qui combinent la température de l'air ambiant et son humidité relative. L'humidité relative de l'air est un facteur de risque intéressant puisqu'un air humide aggrave le stress, voire les dangers liés à la chaleur, en gênant l'évacuation de la vapeur d'eau par la respiration et la sueur. Toutefois, dès qu'il s'agit de chaleurs paroxysmiques, il n'est plus le meilleur discriminant dans le contexte de la Tunisie. Il reste quand même un facteur de stress supplémentaire, surtout pour les classes de chaleur intermédiaires. Outre l'effet de l'humidité, celui du vent est également important comme paramètre de définition des ambiances bioclimatiques, aggravant ou apaisant la sensation de chaleur. En plus, lors des grandes chaleurs, un vent soufflant modérément (brise ou vent léger), peut agir plutôt comme facteur 
apaisant, en facilitant l'évacuation de la sueur. Mais si le vent souffle très fort, il devient plutôt un facteur de stress supplémentaire, en activant le dessèchement prononcé de la peau et des muqueuses. Dans ce cas, c'est la valeur absolue de la température qui prévaut comme indicateur de chaleur. En l'occurrence, une journée est qualifiée de très forte chaleur, voire même torride, à partir d'une température supérieure à $40^{\circ} \mathrm{C}$, quelle que soit la vitesse du vent. Météo-Canada, par exemple, réserve l'usage de la vitesse du vent à la quantification du froid en vue de produire des alertes en hiver (www.ec.gc.ca).

\subsubsection{Les seuils " absolus " de froid}

Les statistiques publiées par l'Institut National des Statistiques (www.ins.nat.tn) montrent que dans la ville de Tunis comme partout en Tunisie, le froid coïncide avec le pic principal de la mortalité globale. L'être humain cherche toujours à se protéger contre le froid grâce à des mécanismes physiologiques endogènes thermorégulateurs. Lorsque la température ambiante est basse, les pertes de chaleur augmentent pour un ratio surface corporelle/masse élevé. Ainsi, le grand froid, tout comme la canicule, constitue un danger pour la santé (Besancenot, 1986, 1990). Compte tenu du contexte climato-thermique de la Tunisie, mais également en convenance avec les spécificités physico-thermiques de l'organisme humain, nous proposons une typologie des gammes de froid fondée sur des seuils absolus, représentant la somme des températures quotidiennes maximales diurnes et minimales nocturnes (figure 9).

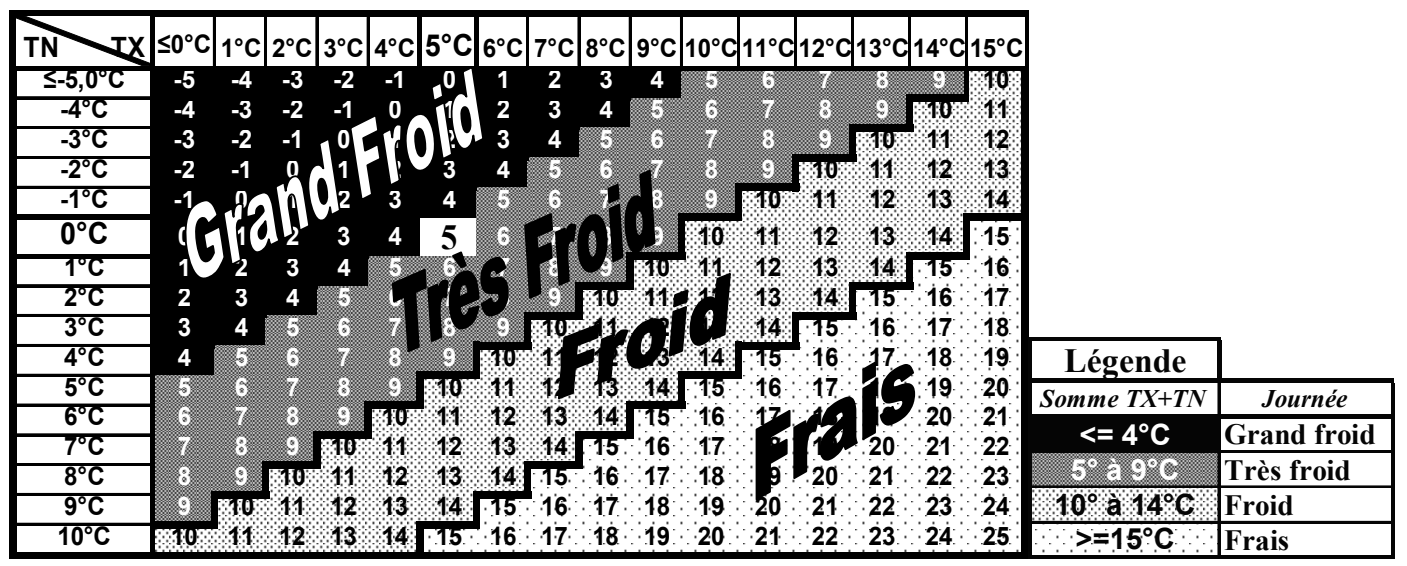

Figure 6 : Indice absolu du froid en Tunisie : seuils et typologie. Absolute index of the cold in Tunisia: thresholds and typology.

Ces seuils s'échelonnent de $5^{\circ}$ en $5^{\circ} \mathrm{C}$, traduisant une typologie simple des niveaux de froid, adaptée au contexte climato-thermique de la Tunisie. Le choix de ces seuils tient compte des principes évoqués plus haut $(\$ 2.2 .1)$, mais il est aussi le fruit de plusieurs expérimentations et également de notre connaissance du climat thermique et de sa perception en Tunisie. Le point central de la grille est le seuil de $0^{\circ} \mathrm{C}$ pour les $\mathrm{TN}$ et $5^{\circ} \mathrm{C}$ pour les TX (soit une somme de $5^{\circ} \mathrm{C}$ ). En dessous de ce seuil, on parle d'un grand froid. Le principe est que chaque augmentation de $1{ }^{\circ} \mathrm{C}$ pour les TX est compensée par une diminution de $1{ }^{\circ} \mathrm{C}$ pour les TN, et vice-versa. Entre un indice (somme TX+TN) de 5 à $9^{\circ} \mathrm{C}$, l'air est jugé très froid. Ensuite, on distingue les catégories d'air moins rigoureux : froid $\left(\right.$ de 10 à $\left.14^{\circ} \mathrm{C}\right)$ et frais $(\mathrm{de} 15$ à $25^{\circ} \mathrm{C}$, avec $\mathrm{TN} \leq 10^{\circ} \mathrm{C}$ et $\mathrm{TX} \leq 15^{\circ} \mathrm{C}$ ).

En somme, dans le contexte d'un pays chaud comme la Tunisie, c'est à partir d'une combinaison entre une température maximale diurne de $15^{\circ} \mathrm{C}$ et d'une température minimale nocturne de $10^{\circ} \mathrm{C}$ qu' on peut juger l'air comme frais, froid, très froid ou de grand froid. Cette typologie est fondée sur la température observée de l'air ambiant. Elle fait abstraction de la température ressentie, exprimée par des indices combinant d'autres paramètres d'ambiance, 
tels que le vent et/ou l'humidité de l'air. En termes de paroxysmes, seuls les jours de grand froid et très froids peuvent être retenus.

\subsection{Comparaison entre les approches statistique et physiologique}

Nous jugeons que chacune des deux approches, statistique « relative» et physiologique « absolue », présente des points forts et des avantages pouvant se compléter. Chacune de ces deux approches peut également répondre à des besoins spécifiques. L'application des seuils relatifs de chaleur et de froid (approche statistique) donne des résultats plus pertinents quant il s'agit d'une analyse minutieuse des paroxysmes thermiques à l'échelle d'une station unique ou d'une région unique. Toutefois, quand il s'agit de comparer un ensemble de stations, appartenant à des régions et des topoclimats différents, ces seuils relatifs posent un problème. En effet, le recours à des seuils statistiques variables d'une région à l'autre, même s'il permet de prendre en considération les conditions d'adaptation, complique l'interprétation et la mise en œuvre des stratégies d'adaptation.

En revanche, les seuils absolus de chaleur et de froid (approche physiologique) se prêtent à une analyse comparative à l'échelle spatiale, entre un ensemble de stations. Leur application présente plusieurs avantages. D'abord, ces seuils se prêtent à une application aisée par le chercheur, mais également par le gestionnaire, le décideur ainsi que tout autre usager, même s'il n'est pas spécialiste. Le recours à des seuils statistiques variables d'une région à l'autre, même s'il permet de prendre en considération les conditions d'adaptation, complique l'interprétation et la mise en œuvre des stratégies d'adaptation. Ensuite, ils tiennent compte de la vocation touristique de la Tunisie. Les millions de touristes qui fréquentent le pays chaque année, en toutes saisons, ont besoin de mises en garde à l'égard de la chaleur et du froid selon les saisons. Il serait aberrant, par exemple, de les alerter d'une chaleur torride à Tunis, avec des TX de $38,5^{\circ} \mathrm{C}$ et de les rassurer d'une « chaleur ordinaire » à Tozeur ou à Kébili, dans le sud du pays, sous prétexte que des $\mathrm{TX}$ de $38,5^{\circ} \mathrm{C}$ et des $\mathrm{TN}$ de $24^{\circ} \mathrm{C}$ correspondent à la « normale » de la saison estivale. Même les habitants de ces régions chaudes méridionales, censés être acclimatés aux conditions thermiques locales, ne manqueraient pas de souffrir et de courir les mêmes risques, à l'air libre, qu'un tunisois voyageant du nord au sud du pays.

Enfin, nous jugeons que la proposition de critères physio-thermiques absolus communs pour toutes les régions du pays, permet une comparaison aisée à l'échelle spatiale, ainsi qu'une évaluation objective des risques liés à la température, dans l'ensemble du pays. Certes, il arrive souvent en Tunisie, particulièrement au cours des saisons intermédiaires, que la température soit caniculaire dans le sud, tandis qu'elle est fraîche dans le nord. Dans ce cas, une personne qui transite du nord au sud du pays et vice-versa, risque des alternances brusques de chaud et de froid, ce qui peut provoquer des problèmes respiratoires. La transition brusque d'une ambiance froide à une autre chaude peut ainsi provoquer un malaise de l'intestin alors qu'une transition brusque du chaud au froid cause des affections de poitrine (Widmaier et al., 2007).

\section{Etude de cas : les paroxysmes thermiques «relatifs» dans la ville de Tunis}

\subsection{Les paroxysmes de chaleur dans la ville de Tunis}

\subsubsection{Fréquence des jours caniculaires dans la ville de Tunis}

Trois catégories de grandes chaleurs relatives ont été mises en évidence jusqu'ici : forte, très forte et torride (figure 4). Seuls les jours de chaleur torride méritent le qualificatif de «paroxysme ». Ceux de très forte chaleur seront évoqués à titre de comparaison. Tous représentent respectivement 86 et 32 jours de l'ensemble de la série observée (1950-2006). Ils 
représentent ainsi 2,3\% de l'ensemble des jours observés d'été ou seulement $0,6 \%$ si on tient compte de l'ensemble des observations quotidiennes interannuelles. Si peu fréquents soientils, ces paroxysmes thermiques marquent la mémoire par leur intensité, par le stress et par les séquelles qu'ils provoquent auprès de toutes les catégories de la population. Il y a cependant une grande variabilité interannuelle d'occurrence des paroxysmes de chaleur (figure 7).

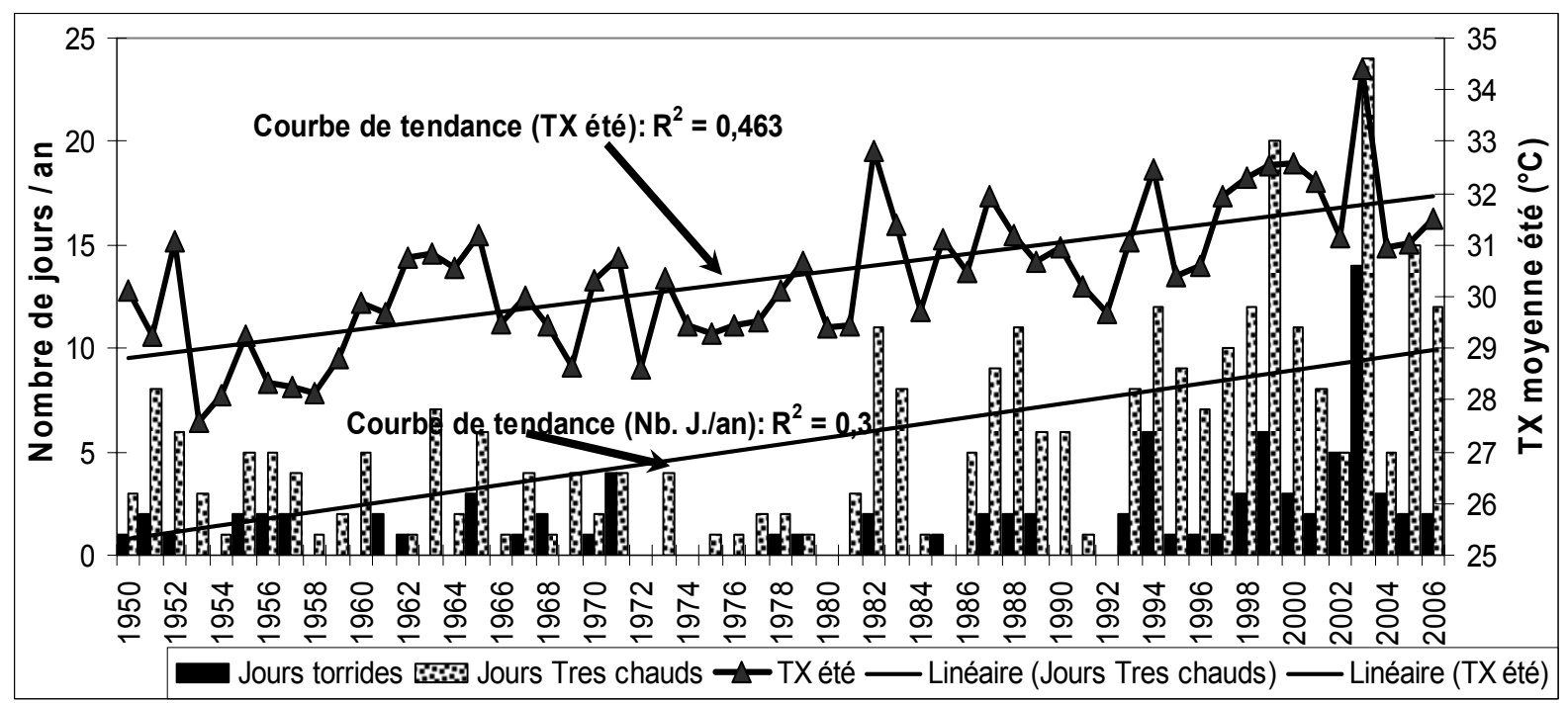

Figure 7: Variabilité interannuelle du nombre de jours de chaleur très forte et torride (1950-2006). Interannual variability of very hot and scorching-hot days (1950-2006).

La fréquence des jours à chaleur très forte et torride affiche une tendance à l'augmentation, particulièrement remarquable depuis le milieu des années 1990. L'année 2003 se distingue par un record de 14 jours torrides et 24 jours de très forte chaleur, auxquels s'ajoutent 14 autres de forte chaleur. En termes de moyenne estivale des TX, c'est également le record $\left(34,4^{\circ} \mathrm{C}\right)$. La fluctuation de fréquence des jours à chaleur paroxysmique est étroitement corrélée aux températures maximales moyennes inter-estivales. En l'occurrence les étés 1982, 1994, 1999 et 2003, particulièrement chauds, enregistrent des fréquences élevées de paroxysmes de chaleur. Une observation plus fine de la fréquence des jours à paroxysmes de chaleur à l'échelle mensuelle, montre des fluctuations encore plus amples (figure 8).

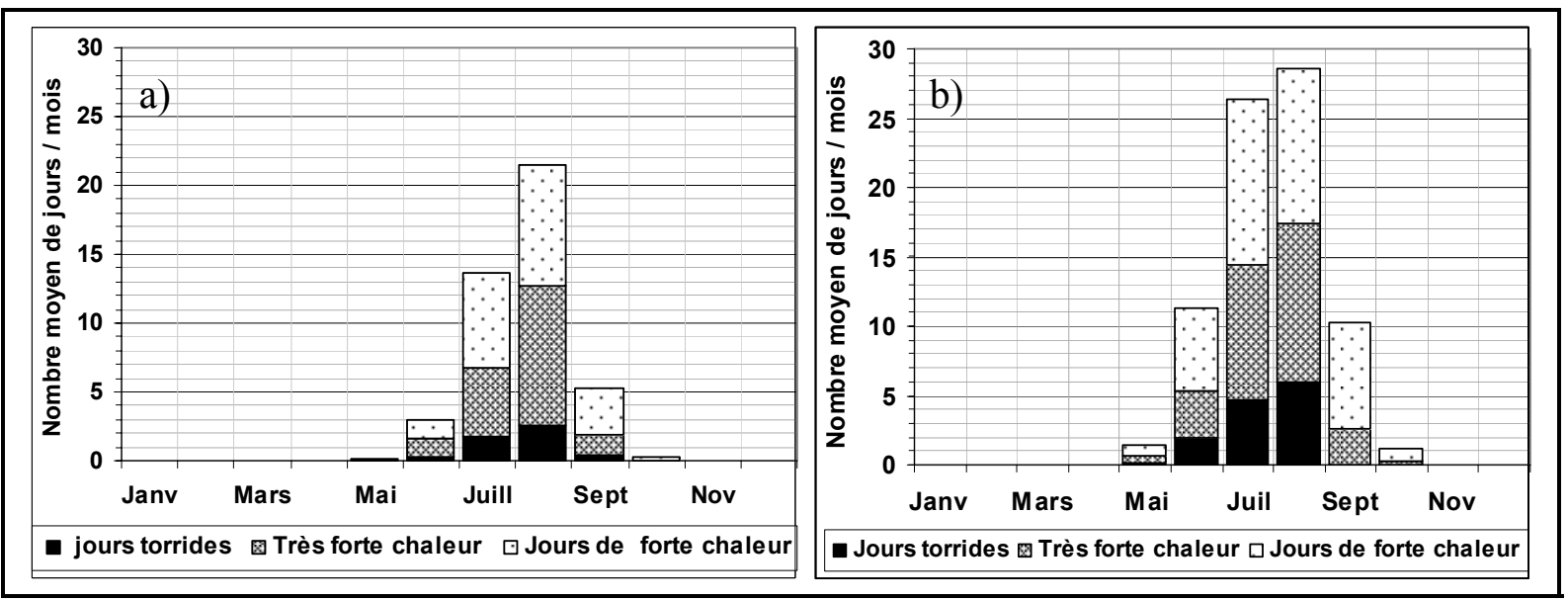

Figure 8 : Répartition mensuelle du nombre de jours de paroxysmes de chaleur (1950-2006) à Tunis-Carthage a) selon la méthode des seuils relatifs, b) selon la méthode des seuils absolus. Monthly distribution of the number of days with peaks of heat (1950-2006) in Tunis-Carthage: a) method of relative thresholds; b) method of absolute thresholds. 
On retrouve un schéma très semblable avec l'application des seuils absolus. D'abord, la saison à risque de grandes chaleurs s'étend sur six mois, allant de mai à octobre. Elle correspond ainsi à la saison estivale, au sens «thermique » (Ben Boubaker, 2009). Toutefois, les jours torrides ont le plus de chance de se produire au milieu et à la fin de l'été (juillet et août). Les deux méthodes de seuils relatifs et absolus de chaleur affichent un régime saisonnier semblable. Toutefois, les seuils relatifs étant plus élevés, ils sous-estiment la fréquence des fortes chaleurs dans la ville de Tunis

A l'échelle quotidienne, on observe une plus grande fluctuation des jours à chaleur paroxysmique (figure 9). Quasiment toutes les années enregistrent des chaleurs caniculaires à Tunis. Il est également fréquent de franchir, le jour, le seuil de $45^{\circ} \mathrm{C}$ dans la ville de Tunis. Quant au record absolu $\left(46,8^{\circ} \mathrm{C}\right)$, il a été enregistré les $01 / 07 / 1998\left(\mathrm{TN}=26,5^{\circ} \mathrm{C}\right)$ et $01 / 08 / 1982\left(\mathrm{TN}=21,6^{\circ} \mathrm{C}\right)$. Toutefois, la seconde date n'a pu être comptabilisée comme journée torride puisque la température minimale observée est inférieure au seuil du $3^{\text {ème }}$ quartile.

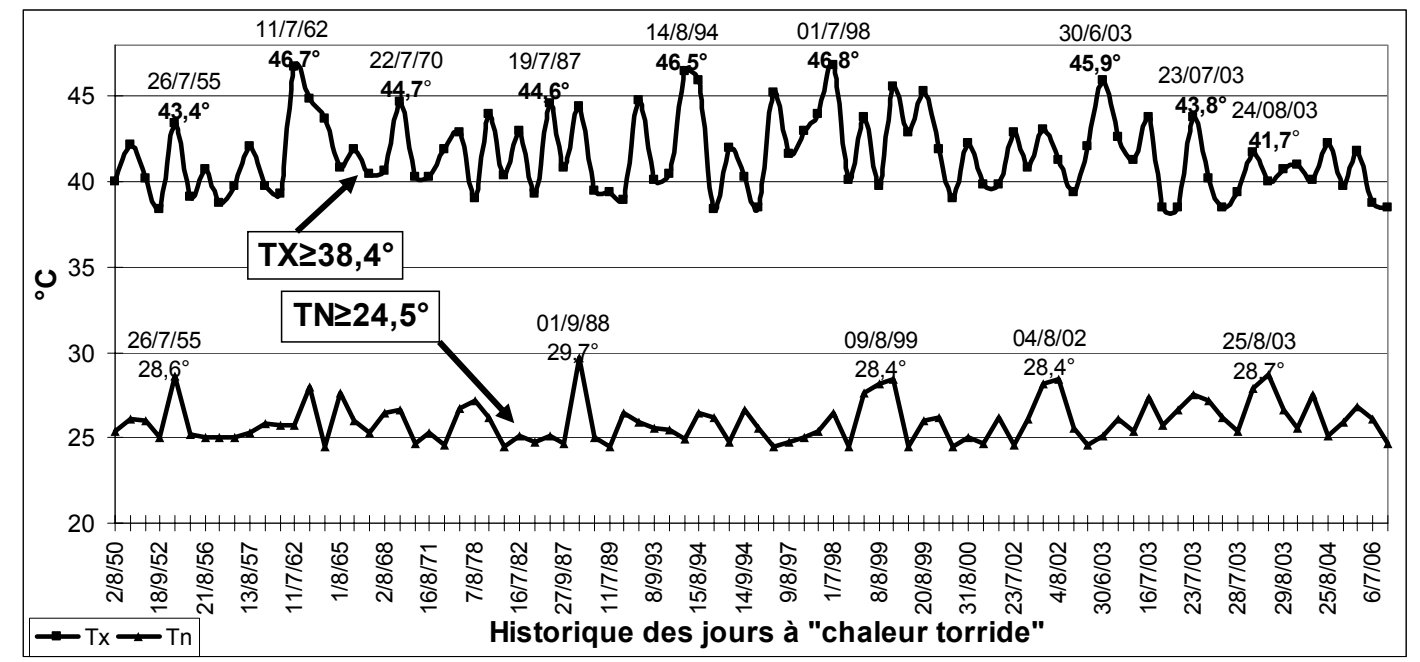

Figure 9 : Paroxysmes de chaleur (jours torrides) observés à Tunis-Carthage : $\mathrm{TX} \geq 38,4^{\circ} \mathrm{C}$ et $\mathrm{TN} \geq 24,5^{\circ} \mathrm{C}$ pour la période 1950-2006 (courbes lissées). Peaks of heat (torrid days) observed in Tunis-Carthage: $T X \geq 38,4^{\circ} \mathrm{C}$ and $T N \geq 24,5^{\circ} \mathrm{C}$ over the period 1950-2006 (smoothed curves).

Il est également intéressant d'effectuer des projections en vue de vérifier les risques de récurrence de ces paroxysmes de chaleur, pour différents temps de retour. A cet effet, la loi Gumbel (dite également loi double exponentielle ou loi des extrêmes) est couramment utilisée pour estimer les temps de retour des valeurs extrêmes de phénomènes climatiques. Cette loi postule que le maximum annuel d'une variable est considéré comme le maximum de 365 valeurs journalières. De ce fait, elle permet de décrire les séries de maxima annuels ${ }^{7}$. Autrement dit, il s'agit principalement d'estimer la probabilité de non dépassement F(xi) qu'il convient d'attribuer à chaque valeur xi. En l'occurrence, la loi Gumbel permet d'effectuer une analyse fréquentielle de la série des TX et des TN maximales et minimales extrêmes observés pour chaque année, pour estimer le temps de retour d'une valeur extrême particulière. Cette prédiction repose sur la définition d'un modèle fréquentiel (équation) permettant de modéliser la probabilité de réapparition d'une valeur donnée des TX ou des TN.

Deux méthodes d'ajustement sont couramment appliquées à la loi Gumbel : la méthode des moments et la méthode graphique.

\footnotetext{
${ }^{7}$ Pour de plus amples détails de la méthodologie Gumbel, consulter en particulier Gumbel (1957), Guillot et Duband (1967), Benkhaled (2007) ou les documents de cours consultables sur http://echo.epfl.ch/e-drologie/chapitres/annexes/.
} 
L'application de cette loi (par méthode graphique) aux températures maximales quotidiennes absolues (TX maximale extrême), à l'échelle de l'année, sur une période d'observation de 58 ans (1950-2007) dans la station de Tunis-Carthage permet d'effectuer l'ajustement de la série des TX (figure 10). Ici, la variable centrée réduite $u$, variable réduite de Gumbel, représente :

$$
\mathbf{u}=\frac{\mathbf{x}-\mathbf{x}_{0}}{\mathbf{G}}
$$

avec $X_{0}$ : paramètre de position de la loi ou encore, le mode ; $G$ : paramètre d'échelle, encore appelé "Gradex» comme "Gradient des valeurs extrêmes» (GUILLOT et DUBAND, 1967). Ce Gradex G peut être aussi considéré comme le descripteur des températures extrêmes.

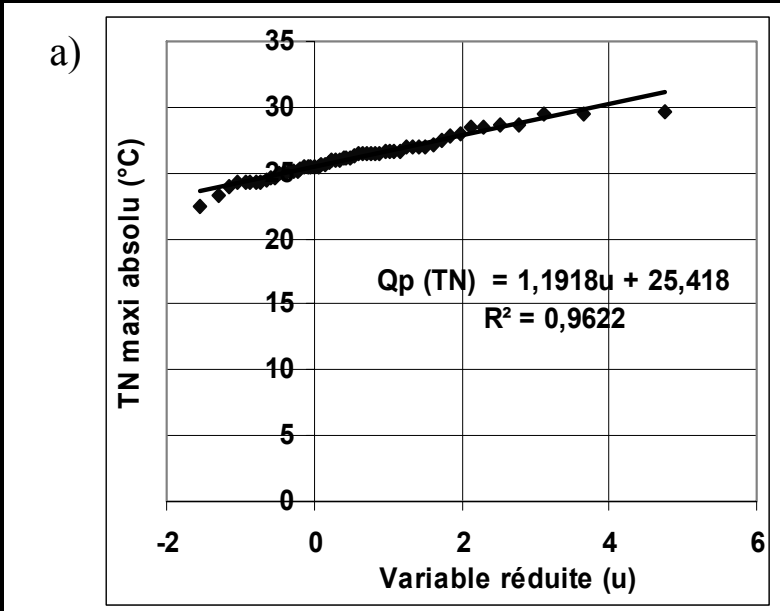

b)

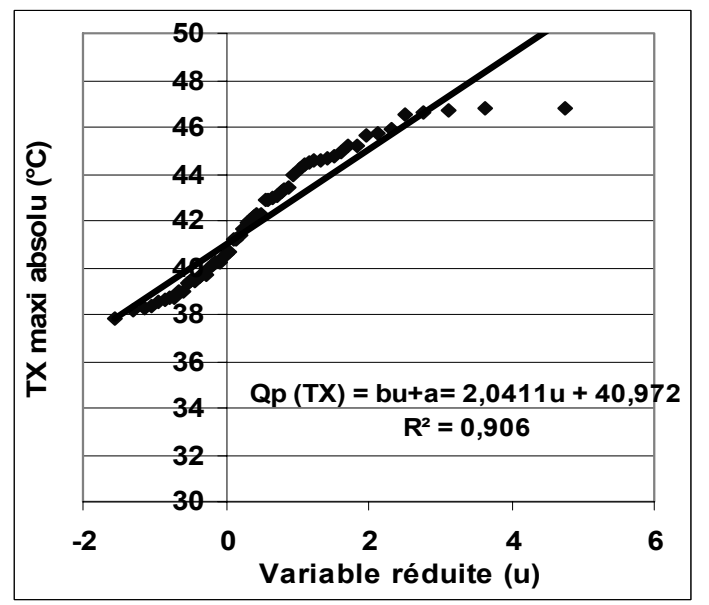

Figure 10: Ajustement de la loi de Gumbel à la série des températures maximales absolues annuelles de Tunis-Carthage (1950-2007): a) pour les TN, b) pour les TX. Adjustment of the Gumbel law to the series Tunis-Carthage (1950-2007) of absolute maximum temperatures: a) for TN, b) for TN.

L'équation de régression de cet ajustement permet de déduire les périodes de retour des paroxysmes des températures maximales diurnes extrêmes (figure 11). Les maxima de températures maximales diurnes (TX) et minimales nocturnes $(\mathrm{TN})$ affichent une certaine régularité d'occurrence. Des TX inférieures à $42^{\circ} \mathrm{C}$ et des TN inférieures à $26^{\circ} \mathrm{C}$ se reproduisent toutes les années. Les températures supérieures à ces deux seuils deviennent ensuite de moins en moins probables.
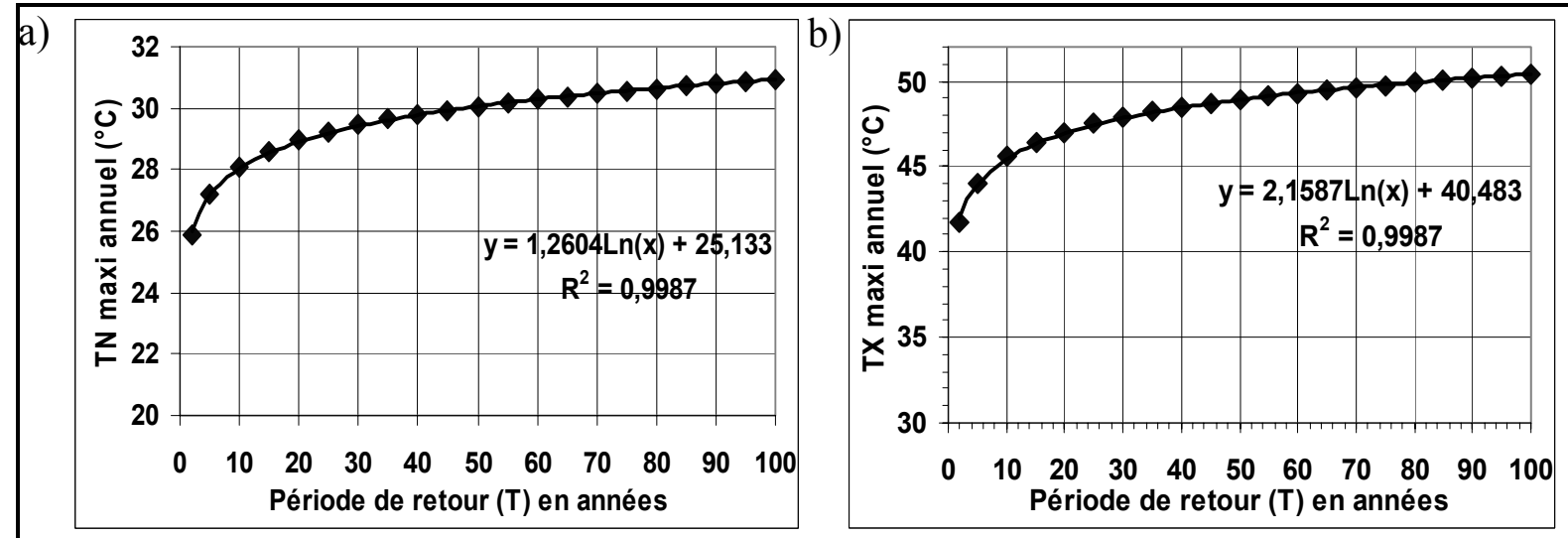

Figure 11 : Durées de retour des températures maximales extrêmes annuelles à Tunis-Carthage (1950-2007). Les projections sont effectuées par la loi de Gumbel : a) avec la série des TN, b) avec la série des TX. Durations of return of the annual extreme maximum temperatures in Tunis-Carthage (1950-2007). Projections are carried out by the Gumbel law with reference to : a) the series of $T N, b$ ) the series of TX. 
Des températures paroxysmiques de l'ordre de $46^{\circ} \mathrm{C}$ pour les $\mathrm{TX}$ et $28^{\circ} \mathrm{C}$ pour les $\mathrm{TN}$ ont une période de retour décennale. Des températures paroxysmiques plus importantes restent possibles, mais avec des probabilités de retour plus espacées, de l'ordre de plus de 20 ans. En l'occurrence, des TX de $49^{\circ} \mathrm{C}$ risquent de se produire tous les demi siècles. Mais, compte tenu de la tendance actuelle au réchauffement thermique observé à la station de Tunis-Carthage, on risque d'avoir des TX frôlant $50^{\circ} \mathrm{C}$ au moins une fois par siècle. Enfin, avec la tendance au réchauffement envisagé au cours de ce siècle, des TX frôlant le seuil de $50^{\circ} \mathrm{C}$ et des TN supérieures à $30^{\circ} \mathrm{C}$ représentent un risque sérieux.

\subsubsection{Intensité des paroxysmes de chaleur dans la ville de Tunis}

L'intensité d'un paroxysme de chaleur dépend de l'ampleur de la chaleur diurne, nocturne, mais également de sa persistance inter-journalière. En effet, une chaleur extrême qui peut être passagère, de 1 à 2 jours, n'a pas le même effet stressant, morbide ou même mortel qu'une autre qui persiste une semaine, voire plus, sans laisser de place à un répit, même nocturne. Pour évaluer l'intensité de la chaleur, nous avons commencé par répertorier tous les jours de chaleur forte, très forte et extrême, c'est-à-dire tous ceux appartenant au $3^{\text {ème }}$ quartile (TX $\geq$ $34,1^{\circ} \mathrm{C}$ et $\mathrm{TN}>22^{\circ} \mathrm{C}$ ). Notre définition des paroxysmes thermiques se limite aux jours torrides tels qu'ils sont définis dans la grille des seuils relatifs (figure 4a). La méthode consiste d'abord à calculer les degrés-jours (DJ) quotidiens, c'est-à-dire (TX - 34, $\left.1^{\circ} \mathrm{C}\right)+(\mathrm{TN}$ $\left.-22^{\circ} \mathrm{C}\right)^{8}$. Ensuite, la somme des degrés-jours de chaque épisode incluant au moins un jour de chaleur torride permet de déduire son poids. Ce poids, divisé par le nombre de jours de l'épisode donne l'intensité de la chaleur du jour $j$.

Par exemple (tableau 5), du 7 au 9 août 1999, trois jours consécutifs à température maximale diurne évoluant entre 39,7 et $45,6^{\circ} \mathrm{C}$ ont été observés. Le DJ du 07/08/1999 se calcule comme suit : $\left(43,8^{\circ}-34,1^{\circ} \mathrm{C}\right)+\left(27,6^{\circ}-22^{\circ} \mathrm{C}\right)=15,3^{\circ} \mathrm{C}$. Les degrés-jours correspondant à l'épisode sont compris entre 11,8 et $17,9^{\circ} \mathrm{C}$. Le poids total de l'épisode se prolongeant sur trois jours équivaut à $45^{\circ} \mathrm{C}$. Ce poids, divisé par trois jours, donne une intensité journalière (I) moyenne de $15^{\circ} \mathrm{C} / \mathrm{j}$.

Tableau 5 : Exemple de trois jours consécutifs enregistrant une chaleur paroxysmique observés à Tunis-Carthage. An example of three consecutive days of paroxysmal heat observed in Tunis-Carthage.

\begin{tabular}{|c|c|c|c|}
\hline Jours & TX & TN & DJ \\
\hline $07 / 08 / 99$ & 43,8 & 27,6 & 15,3 \\
\hline $08 / 08 / 99$ & 39,7 & 28,2 & 11,8 \\
\hline $09 / 08 / 99$ & 45,6 & 28,4 & 17,9 \\
\hline \multicolumn{4}{|c|}{ Poids total en degrés-jour (DJ) $=\mathbf{4 5}^{\circ} \mathbf{C}$} \\
\hline
\end{tabular}

Cette méthode permet la classification des épisodes de chaleur selon leur intensité, leur poids ou leur persistance. Il est alors par exemple possible de repérer les vingt épisodes les plus chauds ayant été observés à Tunis-Carthage au cours de la période d'observation 19502006 (tableau 6). On peut en déduire que les paroxysmes de chaleur surviennent essentiellement au mois de juillet et d'août. Les paroxysmes de chaleur les plus intenses connus dans l'historique de Tunis-Carthage ont été enregistrés au cours des deux dernières décennies. Les étés 1994, 1998, 1999, 2002 et 2003 ont été marqués par des paroxysmes sans précédent. Du point de vue poids, intensité et persistance, c'est le paroxysme du 14 au $17 / 08 / 1994$ qui a été le plus important (avec $\mathrm{P}=50,8^{\circ} \mathrm{C}$ ). Enfin, parmi ces vingt épisodes, l'été 2003 se distingue par les paroxysmes de chaleur les plus récurrents : du 30/06 au 01/07/2003, du 15 au 16/07/2003, du 22 au 25/07/2003 et du 24 au 25/08/2003.

\footnotetext{
${ }^{8}$ D'après le tableau 4 , rappelons que les valeurs $34,1^{\circ}$ et $22^{\circ} \mathrm{C}$ représentent, respectivement, les seuils du $3^{\text {ème }}$ quartile des TX et TN ; les valeurs $23,6^{\circ}$ et $36,7^{\circ} \mathrm{C}$ représentent les seuils respectifs du $9^{\text {ème }}$ décile $\left(90^{\text {ème }}\right.$ centile) des TX et TN de l'été.
} 


\begin{tabular}{|c|c|c|c|c|}
\hline Rang & Episode & $\begin{array}{c}\text { Durée } \\
\text { (en jours) }\end{array}$ & $\begin{array}{c}\text { Poids total } \\
\left(P \text { en }{ }^{\circ} \mathrm{C}\right)\end{array}$ & $\begin{array}{c}\text { Intensité chaleur /jour } \\
\left(\mathrm{I} / \mathrm{j} \text { en }{ }^{\circ} \mathrm{C}\right)\end{array}$ \\
\hline 1 & 14 au $17 / 08 / 1994$ & 4 & 50,8 & 12,7 \\
\hline 2 & 07 au 09/08/1999 & 3 & 45 & 15,0 \\
\hline 3 & 22 au $25 / 07 / 2003$ & 4 & 44,1 & 11,0 \\
\hline 4 & 29 au 01/07/1998 & 3 & 42,4 & 14,1 \\
\hline 5 & 01 au $04 / 08 / 2002$ & 4 & 41,8 & 10,5 \\
\hline 6 & $30 / 6$ au $01 / 07 / 2003$ & 2 & 26,2 & 13,1 \\
\hline 7 & 19 au $20 / 08 / 1999$ & 2 & 26,5 & 13,3 \\
\hline 8 & 01 au $02 / 09 / 1988$ & 2 & 26,4 & 13,2 \\
\hline 9 & 24 au $25 / 08 / 2003$ & 2 & 26,1 & 13,1 \\
\hline 10 & 25 au $27 / 08 / 1971$ & 3 & 25,8 & 8,6 \\
\hline 11 & 15 au $16 / 07 / 2003$ & 2 & 25,7 & 12,9 \\
\hline 12 & $01 \mathrm{au} 02 / 08 / 1968$ & 2 & 20,7 & 10,4 \\
\hline 13 & 07 au 09/08/1997 & 3 & 20,6 & 6,9 \\
\hline 14 & 04 au $05 / 07 / 1993$ & 2 & 20,1 & 10,1 \\
\hline 15 & $04 / 07 / 65$ & 1 & 16,8 & 16,8 \\
\hline 16 & 26 au $27 / 07 / 1996$ & 2 & 16,4 & 8,2 \\
\hline 17 & $11 / 07 / 62$ & 1 & 16,3 & 16,3 \\
\hline 18 & $26 / 07 / 55$ & 1 & 15,9 & 15,9 \\
\hline 19 & 19 au 20/07/1987 & 2 & 15,6 & 7,8 \\
\hline 20 & $22 / 07 / 70$ & 1 & 15,2 & 15,2 \\
\hline
\end{tabular}

Tableau 6 : Les paroxysmes de chaleur « relative » à Tunis-Carthage observés entre 1950-2006 : exemple des 20 épisodes les plus chauds (avec un poids total $\mathrm{P}>15^{\circ} \mathrm{C}$ ). Paroxysms of heat in Tunis-Carthage observed between 1950 and 2006: the 20 hottest periods (total weight $>15^{\circ} \mathrm{C}$ ).

\subsubsection{Répercussions physiologiques des paroxysmes de chaleur dans la ville de Tunis}

Ces paroxysmes de chaleur sont normalement associés au stress (Lamarre et Pagney, 1999). De ce fait, ils représentent un risque pour le confort et la santé des populations exposées. Ce risque incombe à l'agression subie par l'individu, suite au changement brusque de l'ambiance climato-thermique habituelle. L'intégrité physique ou/et mentale de l'individu peut alors être atteinte. La défaillance des systèmes immunitaires peut conduire à l'état morbide, voire la mort de l'individu. Pour mettre en évidence ces risques, nous nous référons, dans un premier exemple, aux indices THI et K calculés pour toutes les observations trihoraires des jours à chaleur torride durant une période d'observation de 37 ans (1970-2006). Toutes les valeurs calculées pour l'indice $\mathrm{K}$, associant température et vitesse du vent, sont négatives. Bien que ces valeurs négatives soient souvent jugées aberrantes et critiquées (cf. $\$ 1.1$ et 1.3), elles traduisent une ambiance qualifiée d'atonique. L'indice THI, associant température et humidité de l'air, a été calculé à $21 \mathrm{~h}, 00 \mathrm{~h}$ et $03 \mathrm{~h}$ pour toutes les nuits ayant atteint ou dépassé un minimum nocturne de $23,6^{\circ} \mathrm{C}$ (seuil D9 pour les TN). Sur l'ensemble des observations quotidiennes, 96,6\% des nuits sont qualifiées de chaudes (THI de 20 à $26,4^{\circ} \mathrm{C}$ ) et $3,4 \%$ des observations appartiennent à la gamme très chaude. Aucun cas de confort n'a été observé. Aucune chance de répit nocturne ne peut donc être associée aux jours à paroxysmes de chaleur. Il va sans dire que ce stress est beaucoup plus intense pendant le jour. Dans $99 \%$ des observations trihoraires quotidiennes interannuelles, correspondant aux jours ayant enregistré des $\mathrm{TX} \geq 36,7^{\circ} \mathrm{C}$ (seuil D9 pour les TX), l'ambiance est qualifiée soit de très chaude, soit de torride. Même en dehors des pics de chaleur en milieu de journée, les habitants de Tunis font face à une ambiance insupportable, qui persiste sans relâche au cours des jours à chaleur paroxysmique.

Le second exemple se réfère à l'été 1994, l'un des plus chauds qu'a connu la ville de Tunis au cours de son histoire récente (figure 12). Il démontre l'effet agressif de la chaleur lors de 
l'épisode le plus intense (poids total $=50,8^{\circ} \mathrm{C}$ et $\mathrm{I}=12,7^{\circ} \mathrm{C} / \mathrm{j}$.) observé au cours des 57 années de référence.

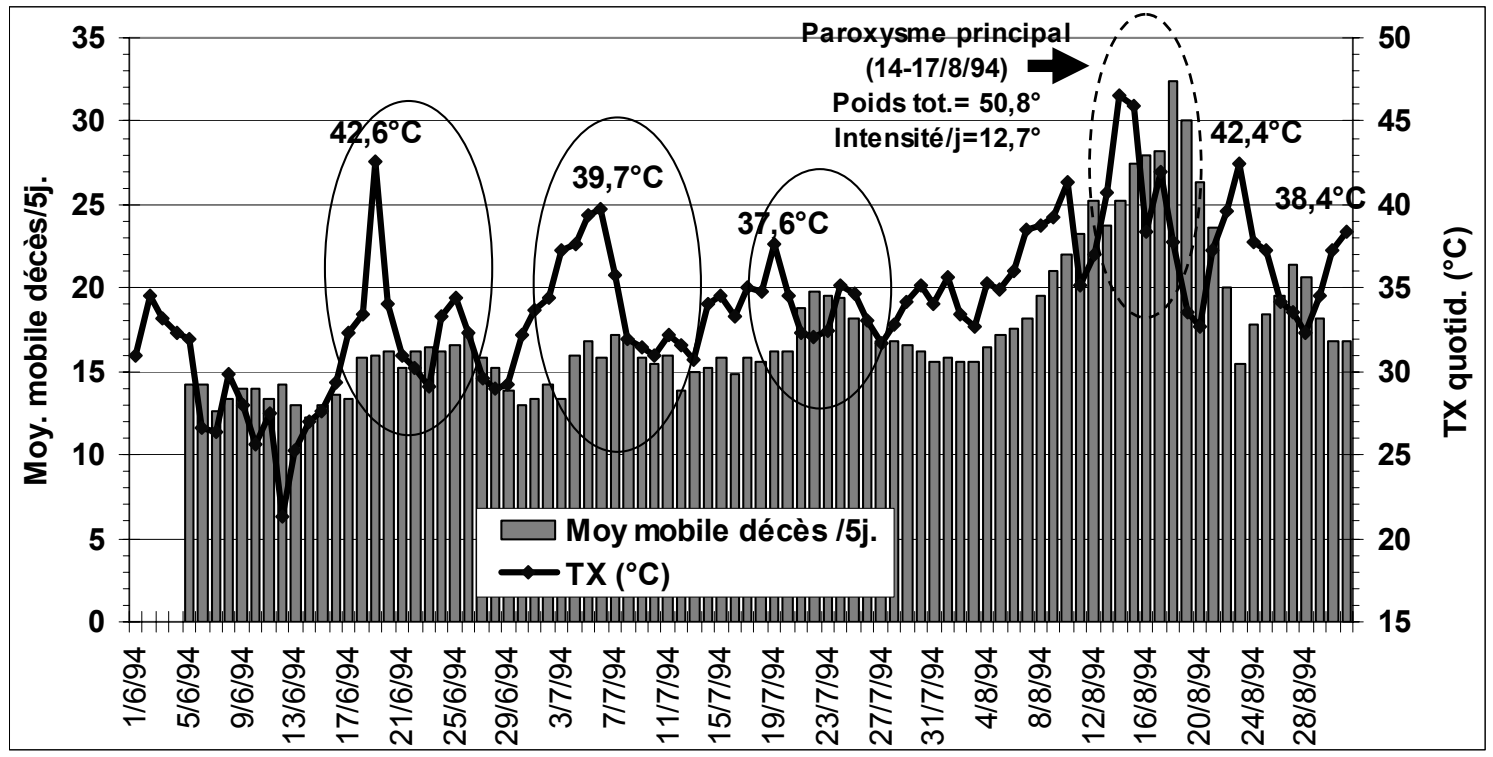

Figure 12 : Moyenne mobile des décès sur cinq jours dans la ville de Tunis et températures maximales diurnes observés à Tunis-Carthage au cours de l'été 1994 (source des données : INM et INS). Shifting deaths average in Tunis over five days and diurnal maximum temperatures observed in Tunis-Carthage during the summer of 1994.

L'effet de ce paroxysme a été mortel provoquant un pic de mortalité, particulièrement apparent de la $2^{\text {ème }}$ à la $3^{\text {ème }}$ semaine du mois d'août. Il a été suivi par un autre moins important vers la fin du mois, suite à une recrudescence de la chaleur. Il est à noter que le paroxysme de chaleur et de mortalité observé vers le milieu du mois d'août a déjà été précédé par au moins trois pics de chaleur (mi-juin, début et fin juillet). Leur effet mortel n'est pas toujours concomitant, mais retardé de quelques jours.

\subsection{Les paroxysmes de froid « relatif » dans la ville de Tunis}

\subsubsection{Fréquence des paroxysmes de froid à Tunis}

Les paroxysmes de froid sont identifiés par référence à la grille du froid relatif décrite plus haut (figure 4). Seuls les jours «très froids » sont jugés paroxysmiques, c'est-à-dire ceux ayant enregistré des TX et TN inférieures au $5^{\text {ème }}$ centile (soit $\mathrm{TN} \leq 3,4^{\circ} \mathrm{C}$ et $\mathrm{TX} \leq 11,6^{\circ} \mathrm{C}$ ). Les jours jugés froids seront évoqués à titre de comparaison. En soumettant les séries de l'ensemble des jours observés entre 1950 et 2006, toutes saisons confondues, à cette grille, on peut recenser :

$-1,6 \%$ des jours qualifiés de frais (soit 323 jours) ;

- 1,2\% froids (soit 246 jours) ;

- seulement $0,5 \%$ de jours très froids ( 94 jours, soit une moyenne de 1,6 jours/an).

La fréquence des jours très froids est très variable d'une année à l'autre (figure 13). L'année 1956 enregistre le nombre record de jours très froids. Par ordre décroissant de fréquence viennent ensuite les années 1963 (7 jours), 1953 (6 jours), 1981 et 1999 (4 jours). En revanche, contrairement aux paroxysmes de chaleur, certaines années n'enregistrent aucun paroxysme de froid. C'est le cas surtout des années 1977, 1989, 1991, 1994, 2001 à 2004, 2006. Au total, on observe une tendance générale à la régression du nombre annuel des jours très froids, particulièrement marquée au cours des deux dernières décennies (figure 13). Par 
ailleurs, l'application des seuils absolus de froid reflète le même profil et la même tendance vers la baisse.

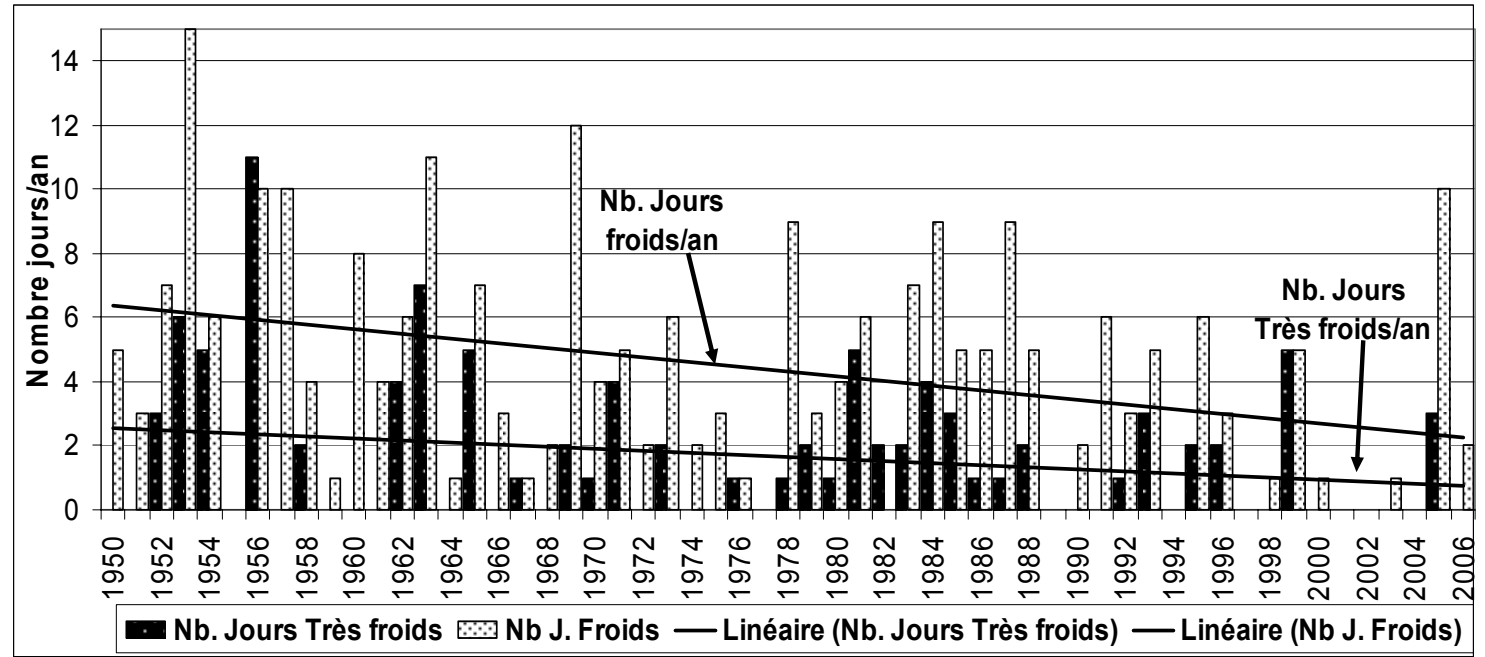

Figure 13 : Variabilité interannuelle du nombre de jours froids et très froids observés à Tunis-Carthage sur la période 1950-2006. Interannual variability of the number of cold and very cold days observed in TunisCarthage (1950-2006).

A l'échelle saisonnière, le milieu et la fin de l'hiver présentent le plus haut risque d'occurrence de paroxysmes de froid à Tunis. Les mois de décembre, de janvier et de février enregistrent la fréquence maximale de jours très froids, mais aussi des jours froids (figure 14). Toutefois, l'occurrence de jours très froids reste possible même en dehors de l'hiver. Ils peuvent être précoces, survenant en novembre, ou tardifs au mois de mars. Dans ces deux cas, par leur effet de surprise, " hors saison », ils représentent les froids les plus redoutés pour le confort et la santé de la population. Cette occurrence est plus fréquente au printemps qu'en automne qui est une saison relativement chaude.
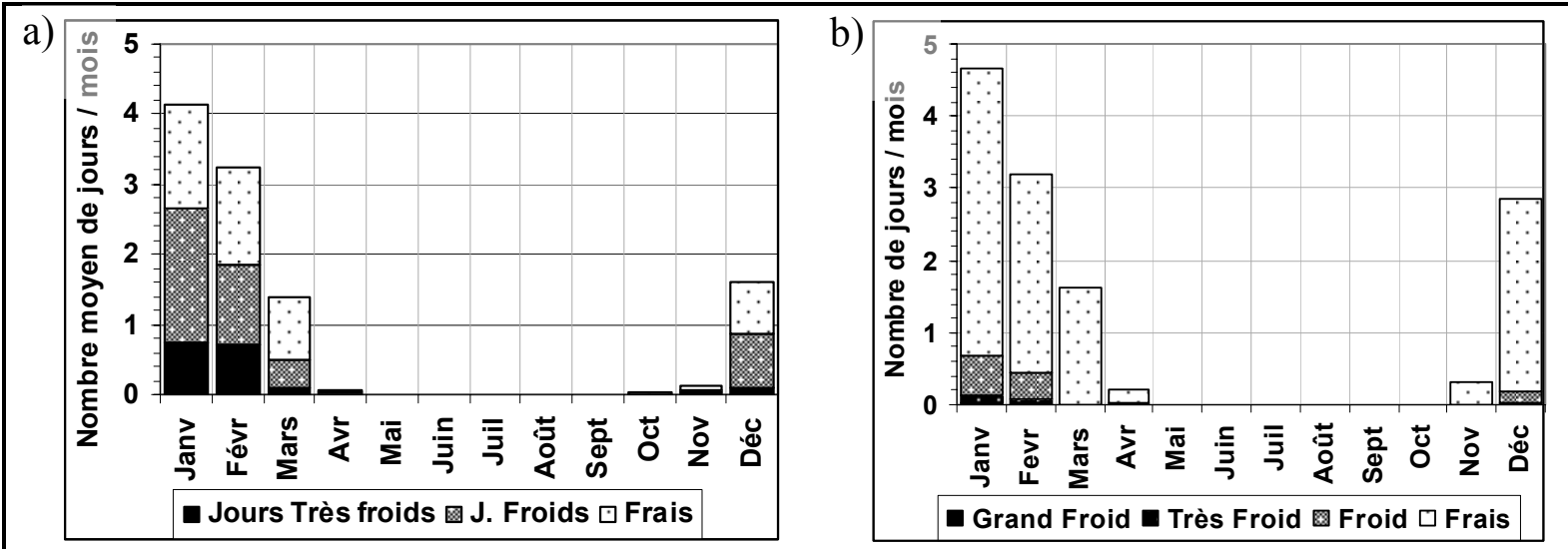

Figure 14 : Fréquence moyenne mensuelle de jours froids à Tunis-Carthage (1950-2006) : a) méthode des seuils relatifs ; b) méthode des seuils absolus. Monthly average frequency of cold days observed in TunisCarthage (1950-2006): a) method of relative thresholds; b) method of absolute thresholds.

En termes de fréquence moyenne totale, les deux méthodes de seuils relatifs et absolus donnent des résultats assez proches. Toutefois, les premiers seuils étant plus bas, ils sousestiment la fréquence totales des jours froids toutes catégories confondues, surtout en décembre et en décembre et en janvier. En revanche, les seuils absolus affichent la 
prédominance des jours frais, qui sont plus caractéristiques de la saison froide à TunisCarthage. Ils témoignent donc d'un froid plus modéré.

Généralement, ces jours de paroxysmes de froid surviennent avec un espacement de plusieurs jours, voire de plusieurs semaines, laissant une période de répit pour la population. Pour évaluer les risques de récurrence de ces paroxysmes de froid, nous avons cherché à modéliser la série des TN minimales extrêmes annuelles ainsi que celle des TX minimales extrêmes observées à Tunis-Carthage (1950-2007). L'ajustement de la loi de Gumbel à ces deux séries annuelles donne de bons résultats, avec des coefficients de détermination respectivement de 0,97 et 0,96 pour les TN et TX (figure 15).
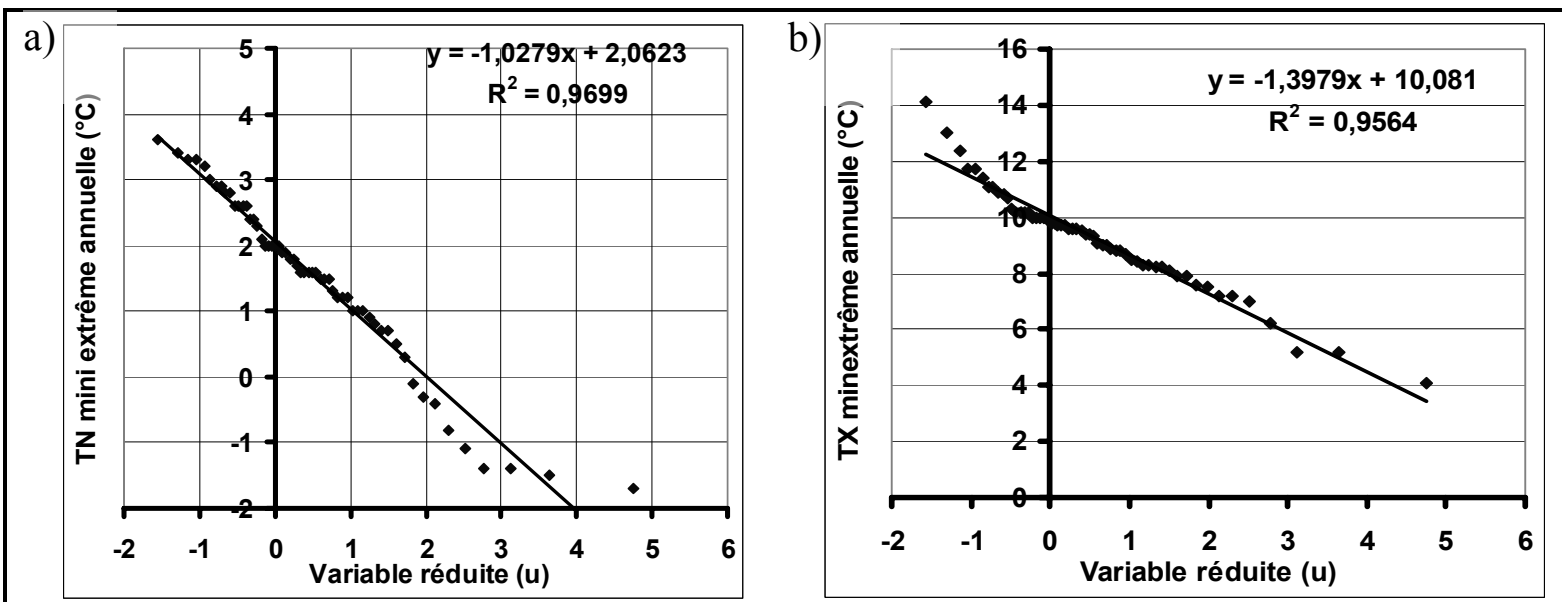

Figure 15 : Ajustement de la loi de Gumbel à la série des températures minimales extrêmes annuelles de Tunis-Carthage (1950-2007) : a) pour les TN; b) pour les TX. Adjustment of the Gumbel law to the TunisCarthage extreme minimum temperature series: a) for $T N$; b) for $T X$.

La validation de ces modèles statistiques permet d'estimer le temps de retour des températures minimales extrêmes nocturnes et diurnes à Tunis-Carthage à l'échelle annuelle. En l'occurrence, des températures négatives nocturnes ont une chance de se reproduire au moins une fois par décennie. Il faut théoriquement attendre un demi siècle environ pour avoir des $\mathrm{TN}$ en deçà de $-2^{\circ} \mathrm{C}$ (figure 16).
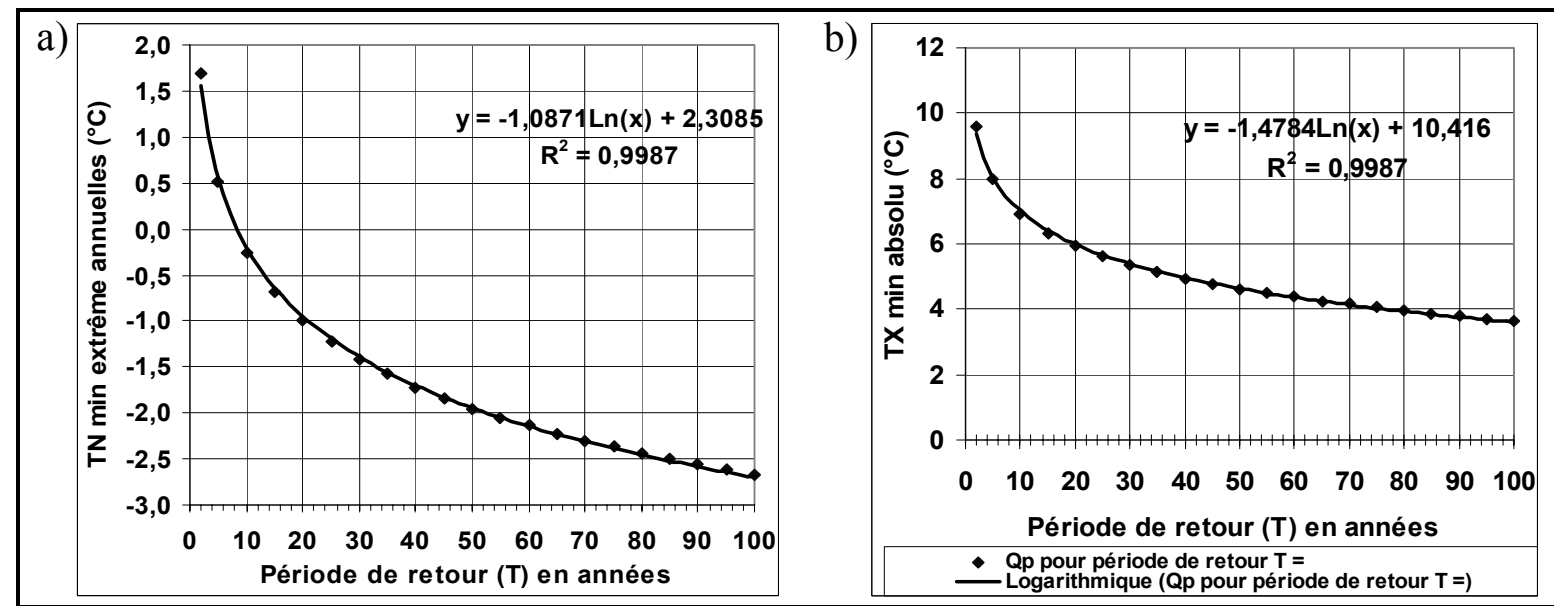

Figure 16: Durées de retour des températures minimales extrêmes annuelles à Tunis-Carthage (les projections sont calculées avec la loi Gumbel, par référence à la série minimales extrêmes annuelles, 19502007) : a) pour les TN ; b) pour les TX. Durations of return of the minimum extreme temperature in Tunis-Carthage (projections are carried out according to the Gumbel law, 1950-2007): a) for TN; b) for TX. 
Les TX minimales extrêmes les plus fréquentes (période de retour inférieure à 5 ans) sont supérieures à $8^{\circ} \mathrm{C}$, ce qui témoigne de la douceur des hivers dans la ville de Tunis (figure $16 \mathrm{~b})$. Des températures maximales diurnes de $1^{\prime}$ 'ordre de $5^{\circ} \mathrm{C}$ ont une chance de se reproduire tous les 30 à 50 ans. Enfin, des TX minimales inférieures à $4^{\circ} \mathrm{C}$ ont une période de retour supérieure à 80 ans. En revanche, des températures négatives diurnes n'ont théoriquement aucune chance de se produire, même après un siècle. A la lumière de ces projections, on peut donc déduire que le climat thermique de la ville de Tunis reste à l'abri des grands froids, semblables à ceux affectant les pays tempérés. D'où la nécessité de concevoir des critères et des seuils appropriés pour la définition des catégories du froid convenant au contexte tunisois.

\subsubsection{Intensité du froid " relatif " dans la ville de Tunis}

Pour évaluer l'intensité du froid, nous prenons en considération deux aspects fondamentaux afférents à la température ambiante. D'abord, l'anomalie thermique, déduite de l'écart entre la température observée et celle de la moyenne du mois correspondant (figure 17). En l'occurrence, le 30 novembre 1980 ne représente pas le jour le plus froid de la série, comme le laisserait penser la figure 17. C'est plutôt le jour le plus froid ayant eu lieu en dehors de l'hiver astronomique. Avec des TX et TN respectives de $8,4^{\circ} \mathrm{C}$ et de $2,4^{\circ} \mathrm{C}$ observées le 30/11/1980, l'anomalie thermique négative calculée par référence à la moyenne $\mathrm{du}$ mois de novembre est respectivement de $-12,3^{\circ}$ et $-9^{\circ} \mathrm{C}$. Un tel froid aussi précoce devrait être plus agressif que s'il avait lieu au milieu de l'hiver.

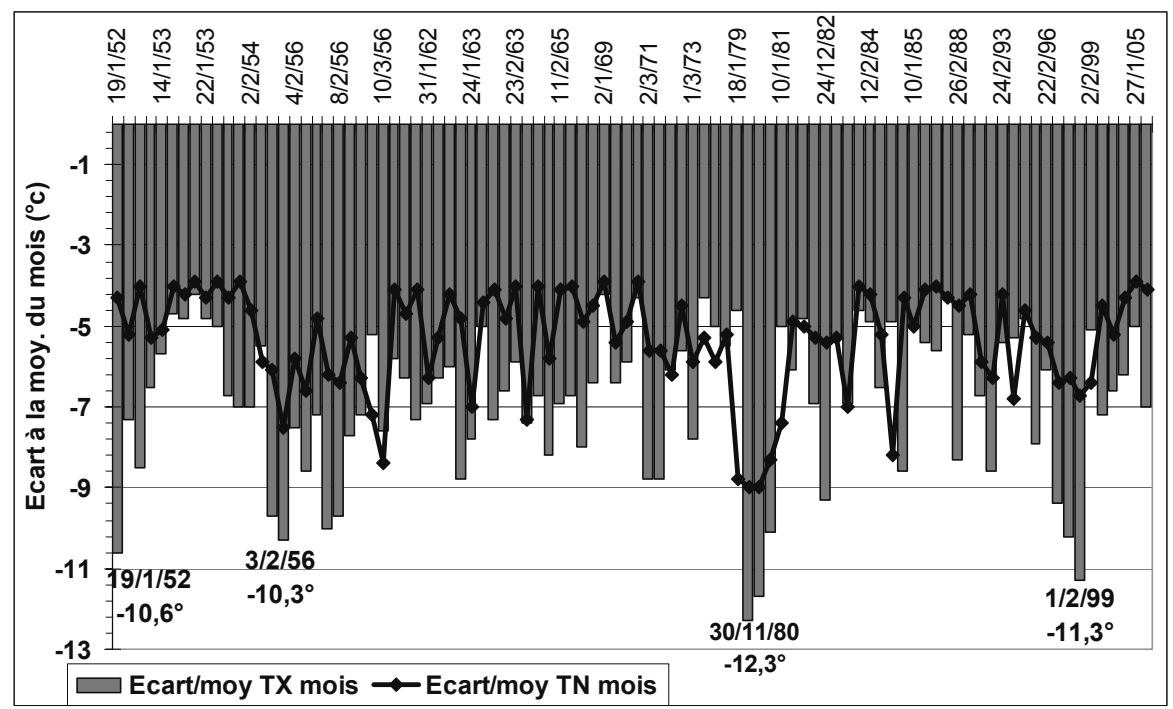

Figure 17 : Ecart à la moyenne mensuelle des TX et des TN correspondant aux jours « très froids » observés à Tunis-Carthage (1950-2006). Monthly average variation of the TX and the TN corresponding to the "very cold" days observed in Tunis-Carthage (1950-2006).

La persistance du froid est également essentielle, étant donné qu'un jour très froid passager n'a pas les mêmes effets physiologiques stressants qu'un froid qui perdure deux jours ou plus. L'étude est menée uniquement sur les jours très froids, bien qu'il arrive souvent que des jours froids ou frais s'intercalent dans un épisode très froid. Pour combiner ces deux aspects révélateurs du froid (valeur de l'anomalie thermique et persistance) et les exprimer par une valeur commune, nous avons d'abord procédé au calcul des degrés-jours (Dj). Il s'agit de calculer l'écart des TX et TN observés par rapport au seuil de leur $5^{\text {ème }}$ centile. Concrètement, il s'agit ici de calculer : $\left[\left(3,4^{\circ} \mathrm{C}-\mathrm{TN}\right)+\left(11,6^{\circ} \mathrm{C}-\mathrm{TX}\right)\right]$, les valeurs 3,4 et 11,6 représentant respectivement le $5^{\text {ème }}$ centile des TN et TX (figures $3 \mathrm{~b}$ et $4 \mathrm{~b}$ ). Ensuite, nous avons calculé le « poids du froid», c'est-à-dire la somme des degrés-jours pour un épisode continu de jours très froids sans interruption (tableau 8 ). 


\begin{tabular}{|c|c|c|c|c|}
\hline Rang & Date de l'épisode & $\begin{array}{c}\text { Poids total } \\
\text { (en degrés-jours) }\end{array}$ & $\begin{array}{c}\text { Durée } \\
\text { (en jours) }\end{array}$ & $\begin{array}{c}\text { Intensité froid/j. } \\
\text { (Iffj, } \text { en }{ }^{\circ} \mathrm{C} / \mathrm{j} \text { ) }\end{array}$ \\
\hline $\mathbf{1}$ & 02 au $08 / 02 / 1956$ & 44,1 & 7 & 6,3 \\
\hline $\mathbf{2}$ & 08 au $10 / 01 / 1981$ & 27,2 & 3 & 9,1 \\
\hline $\mathbf{3}$ & $31 / 01$ au $02 / 02 / 1999$ & 20,1 & 3 & 6,7 \\
\hline $\mathbf{4}$ & 23 au $24 / 01 / 1963$ & 12,2 & 2 & 6,1 \\
\hline $\mathbf{5}$ & 19 au 20/01/1952 & 11,2 & 2 & 5,6 \\
\hline $\mathbf{6}$ & $30 / 01$ au 01/02/1962 & 11,1 & 3 & 3,7 \\
\hline $\mathbf{7}$ & 16 au $18 / 01 / 1979$ & 10,7 & 3 & 3,6 \\
\hline $\mathbf{8}$ & 09 au $11 / 02 / 1965$ & 9 & 3 & 3,0 \\
\hline $\mathbf{9}$ & 20 au $21 / 02 / 1956$ & 8,1 & 2 & 4,1 \\
\hline $\mathbf{1 0}$ & 09 au $11 / 01 / 1985$ & 8,1 & 3 & 2,7 \\
\hline $\mathbf{1 1}$ & 16 au $17 / 02 / 1984$ & 7 & 2 & 3,5 \\
\hline $\mathbf{1 2}$ & $04 / 01 / 1993$ & 6,8 & 1 & 6,8 \\
\hline $\mathbf{1 3}$ & 29 au $30 / 01 / 1963$ & 6,6 & 2 & 3,3 \\
\hline $\mathbf{1 4}$ & 07 au $09 / 01 / 1954$ & 6,5 & 3 & 2,2 \\
\hline $\mathbf{1 5}$ & 13 au $14 / 01 / 1953$ & 6,4 & 2 & 3,2 \\
\hline $\mathbf{1 6}$ & 16 au $17 / 02 / 1965$ & 5,8 & 2 & 2,9 \\
\hline $\mathbf{1 7}$ & $27 / 12 / 1996$ & 5,3 & 1 & 5,3 \\
\hline $\mathbf{1 8}$ & 02 au $3 / 02 / 1954$ & 5,2 & 2 & 2,6 \\
\hline $\mathbf{1 9}$ & $14 / 01 / 1995$ & 5,1 & 1 & 5,1 \\
\hline $\mathbf{2 0}$ & 26 au $27 / 02 / 1988$ & 4,4 & 2 & 2,2 \\
\hline
\end{tabular}

Tableau 8 : Les 20 épisodes les plus froids observés à Tunis-Carthage (poids total $>4,5^{\circ} \mathrm{C}$ ) entre 1950 et 2006. The 20 most cold episodes observed in Tunis-Carthage (total Weight $>4,5^{\circ} \mathrm{C}$ ) between 1950 and 2006.

D'après le tableau 8, on peut déduire que l'année 1956 a connu l'épisode de froid le plus persistant au cours du dernier demi siècle dans la ville de Tunis, avec un poids total de $44,1^{\circ} \mathrm{C}$ au cours de l'épisode du 2 au 8 février. Les températures minimales nocturnes étaient comprises entre $-0,1$ et $2,6^{\circ} \mathrm{C}$, contre 6,2 à $9,3^{\circ} \mathrm{C}$ pour les maximales diurnes. Les écarts à la moyenne de février étaient compris entre $-4,8$ et $-7,5^{\circ} \mathrm{C}$ pour les $\mathrm{TN}$, et $-7,2$ à $10,3^{\circ} \mathrm{C}$ pour les TX. L'année 1981 a enregistré l'épisode de froid le plus intense, en termes de valeurs journalières. L'indice $\left\langle\right.$ If » moyen a atteint $9,1^{\circ} \mathrm{C} / \mathrm{j}$ au cours de l'épisode du 8 au 10 janvier 1981. Cet épisode a enregistré le minimum absolu pour l'ensemble des 57 années d'observation $\left(-1,7^{\circ} \mathrm{C}\right.$ le $\left.8 / 1 / 1981\right)$. Pendant trois nuits consécutives, les TN sont restées négatives (entre $-0,1$ et $-1,7^{\circ} \mathrm{C}$ ), soit des écarts par rapport à la moyenne de janvier de l'ordre de $-7,4$ à $-9^{\circ} \mathrm{C}$. Les TX ont également enregistré de fortes anomalies négatives, de l'ordre de 10,1 à $11,7^{\circ} \mathrm{C}$ les 8 et 9 janvier.

Les deux décennies 1950 et 1960 cumulent plus de la moitié des paroxysmes de froid les plus intenses. En revanche, aucun paroxysme de froid, parmi les plus intenses, n'a été enregistré depuis le début du $21^{\text {ème }}$ siècle. Est-ce une confirmation du réchauffement du climat de la ville de Tunis? L'hypothèse de l'extension urbaine qui fait augmenter l'effet de l'îlot de chaleur est également plausible. La périphérie de l'aéroport de Tunis-Carthage, où se trouve la station météorologique de référence, se trouve en effet envahie par une urbanisation accélérée.

Finalement, pour approfondir l'analyse de l'intensité des paroxysmes de froid à Tunis, nous nous référons à l'échelle trihoraire d'observation des températures, en vue de suivre plus particulièrement la persistance des températures négatives. Il s'avère que le gel, si rare soit-il (6 jours sur l'ensemble de la période d'observation), ne persiste jamais le jour. Les gelées, même quand elles ont lieu, surviennent en fin de nuit (vers 6hTU) et sont brèves.

En somme, le climat thermique tunisois est à l'abri des grands froids. Même les jours jugés « très froids », par référence au climat moyen, représentent un phénomène peu fréquent à 
Tunis. De ce fait, les paroxysmes de froid sont considérés comme un phénomène à risque mineur, beaucoup moins grave que celui des grandes chaleurs. En plus, les intensités enregistrées pour les canicules varient entre 8 et $15^{\circ} \mathrm{C}$ alors qu'elles varient entre $2^{\circ}$ et $9^{\circ} \mathrm{C}$ pour les épisodes très froids.

\subsubsection{Incidences physiologiques des paroxysmes de froid dans la ville de Tunis}

Même si le froid représente un risque mineur dans la ville de Tunis, avec une faible fréquence et une faible intensité, c'est lui qui présente le risque le plus élevé pour la santé et tue le plus de gens. L'effectif moyen de décès calculé pour la période 1991-2007 est de 1600 décès en été, contre 2020 en hiver. Toutefois, il n'est pas aisé d'isoler la part de surmortalité d'origine climatique, surtout que d'autres facteurs peuvent interférer, tels que la pollution atmosphérique, les accidents de circulation ou les épidémies. Focalisons nous, à titre d'exemple, sur l'hiver 1999, l'un des plus froids observés au cours de la période de référence (figure 18).

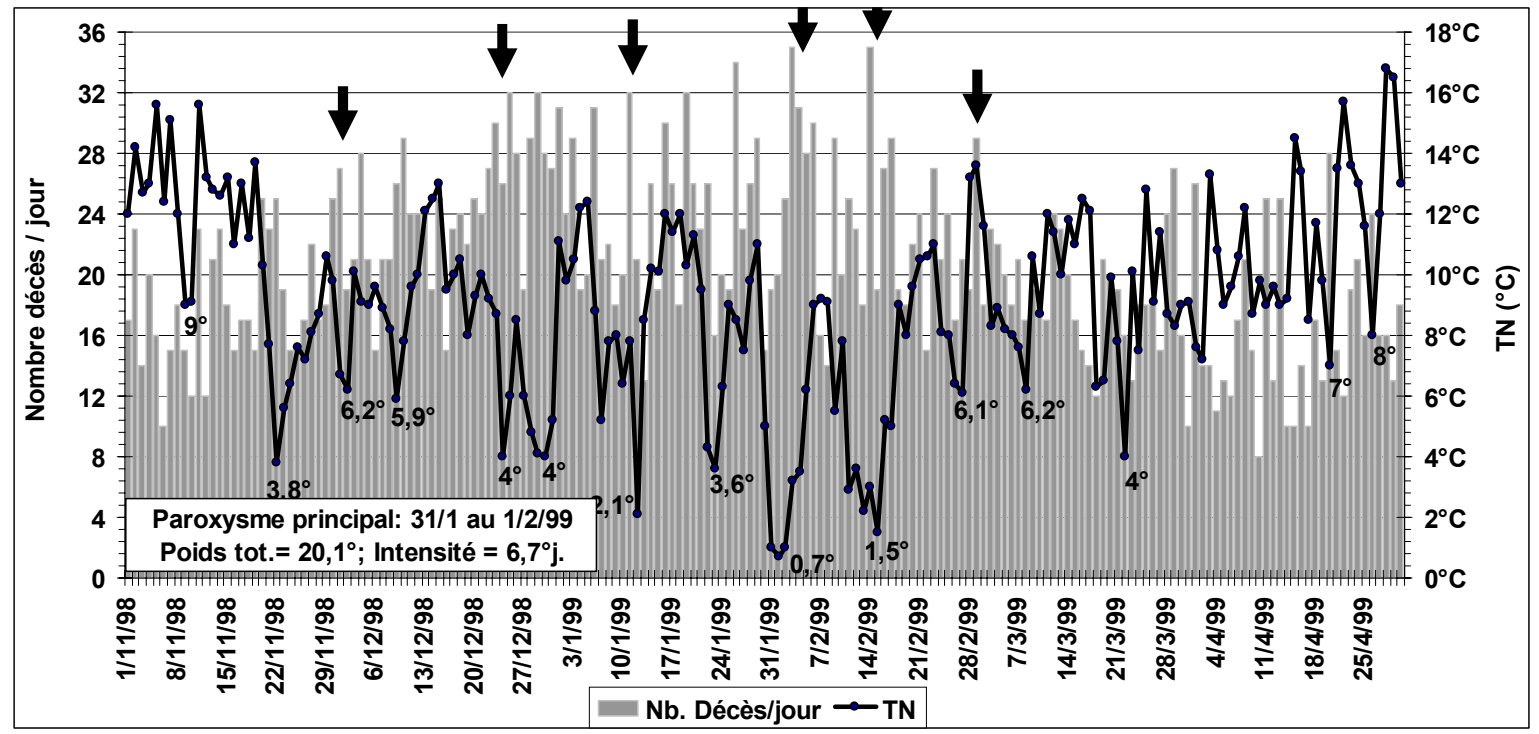

Figure 18 : Moyenne mobile sur 5 jours des décès dans le Grand Tunis et valeurs des TX à la station de TunisCarthage au cours de l'hiver 1998-99 (source des données : INM et INS). Shifting average of the recorded deaths in Larger Tunis and TX at the station of Tunis-Carthage during the winter of 1998-1999.

L'hiver 1998-99 a été marqué par la succession de trois paroxysmes de froid, dont le plus rude a été celui allant du 31/01 au 02/02/1999 (avec un poids total $\mathrm{P}$ de $20,1^{\circ} \mathrm{C}$ et une intensité de $6,7^{\circ} \mathrm{j}$.). Une anti-corrélation entre la courbe des décès et celle des TN minimales quotidiennes est visible : quasiment à chaque chute des TN correspond un pic de mortalité, d'autant plus raide que le froid est vigoureux. Le paroxysme principal de froid est responsable du pic de mortalité le plus important. La semaine suivant, le paroxysme de froid des premiers jours de février a été marquée par une hécatombe : entre 25 et 35 personnes décédées par jour sont enregistrés, contre une moyenne de 20 décès/jour pour l'ensemble de la saison. Les $3 / 4$ de ces décès ont touché la tranche d'âge supérieure à 60 ans. L'effectif des décès provoqués par un tel paroxysme de froid hivernal est plus important que celui induit par un paroxysme de chaleur estivale. Comme le laisse voir également le régime moyen de la mortalité (Henia, 2008), c'est le froid qui est responsable du taux de mortalité le plus élevé dans la ville de Tunis. Donc, un hiver " très froid " ou même " froid » tue beaucoup plus de gens qu'un été «très chaud» ou même «torride ». Il semble que la climato-sensibilité, surtout chez les personnes âgées, soit plus importante en hiver qu'en été (Besancenot, 1990, 2001, 2007 ; Laschewski et Jendritzky, 2002). Le froid agit comme un catalyseur qui précipite une

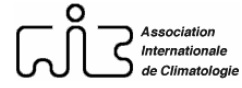


pathologie préexistante (Henia, 2008). L'effet de ces paroxysmes de chaleur et de froid sur la morbidité est plus marqué chez les tranches d'age les plus vulnérables, c'est-à-dire les plus âgés et les plus jeunes (INSP Québec, 2006 ; Fouillet, 2007 ; Rey, 2007).

\section{Conclusion}

La définition des paroxysmes thermiques et de leurs critères d'identification en Tunisie se réfère impérativement au contexte climato-thermique du pays. Les modèles appliqués ailleurs, particulièrement dans les pays tempérés et froids, ne peuvent être transposés intégralement à ce contexte. Ils doivent être maniés avec prudence ou subir des rectifications. Dans ce travail, deux méthodes d'approche ont donc été proposées pour définir et identifier les paroxysmes de chaleur et de froid. La première, testée pour la ville de Tunis, définit des seuils de chaleur et de froid relatifs. Elle se réfère aux températures vécues in situ, sur une longue période. Cette méthode est particulièrement pertinente car elle prend en considération les conditions d'adaptation au climat thermique local. Ces seuils relatifs sont donc plus pertinents quand il s'agit de mener une étude ponctuelle des paroxysmes thermiques, comme c'est le cas pour la ville de Tunis. La seconde se réfère à des seuils absolus de chaleur et de froid. Ces derniers se prêtent bien à une investigation à l'échelle de l'ensemble du pays. Ils permettent de comparer les différentes stations et de dégager les nuances régionales.

Les paroxysmes de chaleur, comparés à ceux de froid, représentent par leur fréquence, leur intensité et leur persistance, le risque thermique le plus stressant pour le confort et la santé de la population tunisoise. Avec la tendance au réchauffement du climat global, ces paroxysmes risquent de s'amplifier au cours du siècle, comme en témoignent les modèles de prévision. Cela va de pair également avec les prévisions du GIEC qui envisagent, dans le cadre des changements climatiques, à la fois une augmentation de la température et un accroissement de la fréquence et de l'intensité des paroxysmes (Besancenot et Laaidi, 2002 ; IPCC, 2007).

En revanche, les paroxysmes de froid, bien qu'ils soient moins fréquents et moins persistants que ceux liés à la chaleur, représentent un aléa plus morbide, voire plus mortel (Besancenot et Laaidi, 2002; Henia, 2008; Ben Boubaker, 2010). Cet aspect, qui semble paradoxal, mérite une investigation plus approfondie. Une échelle de vigilance ainsi que des stratégies d'adaptation pertinentes, tenant compte de ces deux types de paroxysmes, sont également impératives pour la ville de Tunis en particulier, et la Tunisie en général. Le choix de l'une ou de l'autre des deux méthodes proposées dans ce travail peut être utile à cet effet. Nous jugeons que les seuils relatifs conviennent pour des investigations ponctuelles, tenant compte du climat moyen local. Les seuils absolus conviennent pour une échelle plus large, en l'occurrence la Tunisie entière. Ils permettent de mieux appréhender les seuils de risques thermiques extrêmes et d'en saisir les nuances régionales. Ces seuils absolus ont des références stables, facilitant l'analyse approfondie des paroxysmes thermiques dans leur variation spatio-temporelle. Il s'avère également indispensable de développer une approche pertinente pour l'estimation des températures ressenties, tenant compte des autres facteurs d'ambiance (vent et humidité de l'air) et d'en déduire des seuils de risque de chaleur et de froid appropriés pour la Tunisie.

\section{Bibliographie}

ALOUANE T., 2002 : Les ambiances climatiques dans les principales régions touristiques de la Tunisie. Thèse de Doctorat (en arabe), Université de Tunis, FSHS, 470 pages. 
BEN BOUBAKER H., 2006 : Chaleur et canicules estivales dans les principales villes côtières de Tunisie. Publications de l'Association Internationale de Climatologie, 19, 107112.

BEN BOUBAKER H., 2009 : Fortes chaleurs et topoclimat thermique à Siliana (Tunisie tellienne). Géographie et Développement, 18, 65-92.

BEN BOUBAKER H., 2010 : Les paroxysmes de chaleur et de froid dans la ville de Tunis : étude de cas extrêmes. Revue Tunisienne de Géographie, 41, accepté.

BEN BOUBAKER H., HENIA L. et BENZARTI Z., 2004 : Circulations méridiennes et extrêmes pluvio-thermiques en Tunisie. Actes du $17^{\text {ème }}$ colloque de l'AIC, Caen, 4 pages.

BENKHALED A., 2007 : Distributions statistiques des pluies maximales annuelles dans la région du Chelif. Comparaison des techniques et des résultats. Courrier du Savoir, 8, 83-91.

BESANCENOT J.-P., 1986 : Réflexions sur le pouvoir réfrigérant du vent dans les montagnes Nord-Méditerranéennes. Revue de Géographie Alpine, 74(1-2), 11-20.

BESANCENOT J.-P., 1990 : Climat et tourisme. Editions Masson, 223 pages.

BESANCENOT J.-P. 1992 : Risques pathologiques: rythmes et paroxysmes climatiques. Editions John Libbey, 413 pages.

BESANCENOT J.-P., 1997 : Les grands paroxysmes climatiques et leurs répercussions sur la santé. Presse therm. Clim., 134, 237-246.

BESANCENOT J.-P., 2001 : Climat et santé. Paris, PUF, Collection médecine et société, 128 pages.

BESANCENOT J.-P., 2002 : Vagues de chaleur et mortalité dans les grandes agglomérations urbaines. Environnement Risques Santé, 1, 229-240.

BESANCENOT J.-P., 2007 : Notre santé à l'épreuve du changement climatique. Editions Delachaux et Niestlé, 222 pages.

BESANCENOT J.-P. et LAAIDI K., 2002 : Mortalité en France selon le contexte thermique : réalité présente et scénarios pour le XXI'me siècle. Séminaire de restitution : programme gestion et impacts du changement climatique, Toulouse, MATE et Médias-France, 81-83.

ESCOURROU P., 1994 : La bioclimatologie humaine de Tunis-Carthage en janvier et juillet 1991. In Variabilité du climat et stratégies d'adaptation humaine en Tunisie. Publication de l'Université de Tunis, 165-178.

EUROWINTER GROUP, 1997 : Cold exposure and winter mortality from ischæmic heart disease, cerebrovascular disease, respiratory disease, and all causes in warm and cold regions of Europe. Lancet, 349, 1341-1346.

FOUILLET A., 2007 : Surmortalité liée aux vagues de chaleur : modélisation des variations spatio-temporelles de la mortalité générale en fonction des caractéristiques du climat. Thèse de Doctorat, Université Paris XI, 246 pages.

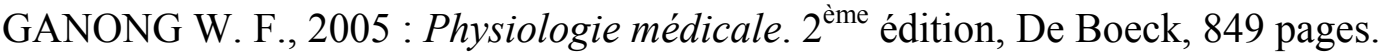

GUILLOT P. et DUBAND D., 1967: The Gradex to compute extreme flood probability from the observation rainfall. International hydrology symposium. Vol. 1, Colorado State University, Fort Collins, USA, 506-515.

GUMBEL E. J., 1957 : Méthodes graphiques pour l'analyse des débits de crues. Revue de Statistique Appliquée, 5(2), 77-89.

HENIA L., 2008 : Climat et mortalité en Tunisie. Bioclimatologie et Topoclimatologie. Publication de la Faculté des Lettres, des Arts et des Humanités de Manouba, 173-182. 
HENIA L. et ALOUANE T., 2007: Le potentiel climato-touristique de la Tunisie. Publications de l'Association Internationale de Climatologie, 20, 27-33.

HENIA L. et ALOUANE T., 2009 : Les ambiances caniculaires dans les villes tunisiennes : cas de Monastir, Kairouan et Tozeur. Geographica Technica, numéro spécial, 241-246.

INSTITUT DE VEILLE SANITAIRE, 2003 : Impact sanitaire de la vague de chaleur en France survenue en août 2003. INVS, Département des maladies chroniques et traumatismes, Département Santé Environnement, 75 pages, consultable sur: www.invs.sante.fr/publications/2003/chaleur_aout_2003/rap_chaleur_290803.pdf.

INSTITUT DE VEILLE SANITAIRE, 2004 : Froid et santé. Eléments de synthèse bibliographique et perspectives. Rapport d'investigation, 48 pages.

INSTITUT NATIONAL DE SANTE PUBLIQUE DU QUEBEC, 2006 : Effets du climat sur la mortalité au Québec méridional de 1981 à 1999 et simulations pour des scénarios climatiques futurs. Rapport interne, 95 pages.

IPCC, 2007 : Fourth Assessment Report: Climate Change 2007: The Physical Science Basis. Edited by Rajendra K. Pachauri, IPCC Chairman, Andy Resinger, Head of Technical Support Unit, The Core Writing Team, IPCC, Geneva, Switzerland, 18 pages.

KEATINGE W. R., DONALDSON G.C, CORDIOLI E, MARTINELLI M., KUNST A. E., MACKENBACH J. P., NAYHA S. et VUORI I., 2000 : Heat related mortality in warm and cold regions of Europe: observational study. British Medical Journal, 321, 670-673.

KUNST A. E, 2001 : Refroidissement éolien et mortalité aux Pays-Bas : Climat et Santé. Cahiers de bioclimatologie et de biométéorologie humaine, 63-73.

LAMARRE D. et PAGNEY P., 1999 : Climats et sociétés. Editions A. Colin, 272 pages.

LAAIDI K., PASCAL M., BERAT B., STRAUSS B., LEDRANS M. et EMPEREURBISSONNET P., 2006 : Système d'alerte canicule et santé 2006. Rapport opérationnel, Institut de Veille Sanitaire, Saint-Maurice, 46 pages.

LASCHEWSKI G. et JENDRITZKY G., 2002 : Effect of the thermal environment on human health: an investigation of 30 years of daily mortality data from SW Germany. Climate Research, 21, 91-103.

MARTIN C., RIOU B. et VALLET B. (sous la direction de), 2006 : Physiologie humaine appliquée. Editions Arnette, 1099 pages.

OSCZEVSKI R., 1995 : The basis of Wind Chill. Arctic, 48, 4, 372-382.

REY G., 2007: Surmortalité liée aux vagues de chaleur: facteurs de vulnérabilité sociodémographique et causes médicales des décès. Thèse de Doctorat, Université Paris XI, 206 pages.

ROBINSON P.-J., 2000 : On the definition of heat wave. Journal of Applied climatology, 40, 762-774.

STEADMAN R. G., 2001 : La limite inférieure de la température apparente : élaboration d'une échelle fiable pour le refroidissement éolien. Cahiers de bioclimatologie et de biométéorologie humaine, 51-62.

TREMBLAY M., 2003 : Du refroidissement éolien et du facteur humidex (le ridicule a une température). Document numérique consulté sur : http://ptaff.ca/humidex/.

VIGNEAU J.-P., 2000 : Géoclimatologie. Editions Ellipses, 334 pages.

WAHL L., PAUL P., PICHARD C., MORY N., DROGUE G. et PFISTER L., 2005 : Les canicules de l'été 2003 : un événement météorologique exceptionnel dans le quart nord-est de la France. Revue Géographique de l'Est, 2, 67-77. 
WIDMAIER E. P., RAFF H. et STRANG K. T., 2007: Physiologie humaine: les mécanismes du fonctionnement de l'organisme. $4{ }^{\mathrm{ème}}$ éditions française, Editions Maloine, 809 pages.

\section{Autres documents consultés sur internet :}

http://www.infoclimat.fr/stations-meteo/expli_humidex.php (dernière date de consultation : 15/09/2009).

http://www.ins.nat.tn (dernière date de consultation : 05/06/2010).

http://www.meteosuisse.admin.ch/web/fr/meteo/actualite_meteo/Tropicales.html (dernière date de consultation : 05/06/2010).

http://pagesperso-orange.fr/macon71-meteo/index_fichiers/Page583.htm (dernière date de consultation : 05/06/2010). 